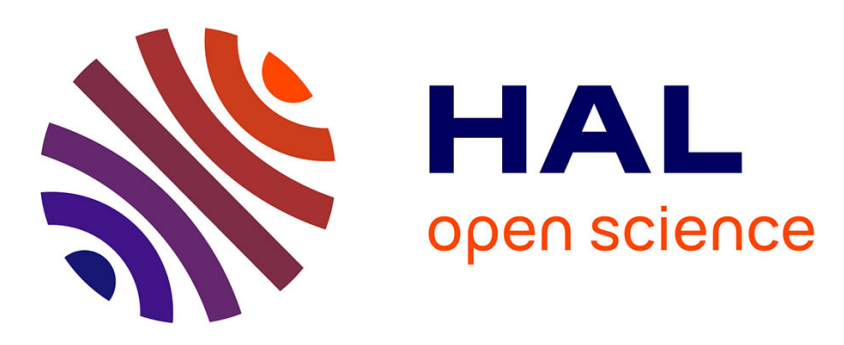

\title{
Random sampling remap for compressible two-phase flows
}

Mathieu Bachmann, Philippe Helluy, Jonathan Jung, Hélène Mathis, Siegfried Müller

\section{- To cite this version:}

Mathieu Bachmann, Philippe Helluy, Jonathan Jung, Hélène Mathis, Siegfried Müller. Random sampling remap for compressible two-phase flows. Computers and Fluids, 2013, 86, pp.275-283. 10.1016/j.compfluid.2013.07.010 . hal-00546919v2

\section{HAL Id: hal-00546919 https://hal.science/hal-00546919v2}

Submitted on 4 Mar 2014

HAL is a multi-disciplinary open access archive for the deposit and dissemination of scientific research documents, whether they are published or not. The documents may come from teaching and research institutions in France or abroad, or from public or private research centers.
L'archive ouverte pluridisciplinaire HAL, est destinée au dépôt et à la diffusion de documents scientifiques de niveau recherche, publiés ou non, émanant des établissements d'enseignement et de recherche français ou étrangers, des laboratoires publics ou privés. 


\title{
Random sampling remap for compressible two-phase flows
}

\author{
M. Bachmann, P. Helluy, J. Jung, H. Mathis and S. Müller \\ RWTH Aachen and IRMA, Université de Strasbourg
}

\begin{abstract}
In this paper we address the problem of solving accurately gas-liquid compressible flows without pressure oscillations at the gas-liquid interface. We introduce a new Lagrange-projection scheme based on a random sampling technique introduced by Chalons and Goatin in [CG07]. We compare it to a ghost fluid approach introduced in [WLK06, MBKKH09] which is based on the ghost fluid method for the poor [AK01]. Despite the non-conservative feature of the schemes, we observe the numerical convergence towards the relevant weak solution for shock-contact interaction test cases. Finally, we apply the new scheme to the computation of the oscillations of a spherical air bubble inside water.

Keywords: Finite volume, Godunov scheme, Ghost fluid method, Lagrange-projection, Glimm scheme, bubble oscillations.
\end{abstract}

\section{Introduction}

The bad precision of conservative Godunov schemes applied to two-fluid flows is a subject that has been studied now for more than twenty years, see [Abg88, Kar94, SA99a, BHR03, MBKKH09] and included references. This bad precision implies perturbations on the pressure profiles, that are often called the "pressure oscillations" phenomenon.

For the moment, it has not been possible to design a simple conservative

Email address: helluy@math.unistra.fr (M. Bachmann, P. Helluy, J. Jung, H. Mathis and S. Müller)

Preprint submitted to Elsevier

September 5, 2012 
scheme that preserves the constant velocity-pressure states. This property, which amounts to preserving the contact discontinuities in one-dimensional flows, seems to be mandatory for obtaining reliable schemes. Thus, many authors have proposed modified Godunov schemes in order to achieve this property. Karni [Kar94] proposes to solve the pressure evolution equation instead of the mass fraction evolution equation at the interface. Abgrall and Saurel [Abg88, SA99a] propose to solve the mass fraction equation in a non-conservative way in order to recover the preservation of constant velocity-pressure states (S-A approach). Fedkiw and collaborators [FAMO99] introduced the ghost fluid method (GFM): at the interface, they propose to introduce two virtual fluids in order to construct a scheme that only requires a one-fluid Riemann solver. The GFM has been improved in many works. We will concentrate here on one variant of the GFM based on the ghost fluid method for the poor [AK01, FRS08, WLK06, MBKKH09]. It is not possible to give a comprehensive survey of this field, but many other attempts have been proposed: changing the two-fluid model to a more general one [SA99b, WK05, KL10], using a Lagrangian approach at the interface [HMM08], etc.

A common feature of all the above-mentioned approaches is that the schemes are generally non-conservative. It is possible to construct very exotic schemes that are conservative but they are then very complicated and can be used only for academic test cases [HMM08]. A natural question arises, which is whether these schemes converge, or not, towards the relevant solution of the initial twofluid models. Indeed, generally, non-conservative schemes converge towards wrong solutions. It is a purely nonlinear behavior [HL94], which is still not yet well understood because a non-conservative Lax-Wendroff theory does not exist (for a recent work on this aspect, see [AK10]). Here, the situation is rather subtle because the non-conservation of the schemes is generally located at the contact discontinuity, which is a linearly degenerated field. When all the discontinuous waves (shocks and contacts) are well separated, it is thus not a full paradox to observe convergence towards the good solution. However, in case of complicated nonlinear interactions, when all the waves are mixed, it is diffi- 
cult to understand why the non-conservative approach leads to well converging schemes. For one-dimensional problems, wave mixing occurs in very simple situations: at the initial time of a Riemann problem, for instance, or when a shock wave is running over a moving interface.

Our first objective in this paper is to provide a new non-conservative scheme for solving two-fluid flows. Our approach is an adaptation of previous works of Goatin, Chalons and Coquel [CG07, CC08] on Lagrange-projection schemes. The idea is to use a projection step based on random sampling techniques, very similar to the Glimm scheme method. The classical Glimm scheme [G165] implies an exact Riemann solver. Here, because the random sampling is only performed in the projection step, it is possible to rely on approximate Riemann solvers in the Lagrange step. We will see that in the presence of strong shocks, our approach has to be adapted, in order to avoid oscillations and nonconvergence: we simply propose to perform the random sampling strategy only at the two-fluid interface. As for other schemes, our random sampling projection scheme is not conservative. Let us recall that the Glimm scheme is not conservative too, but possesses statistically conservation properties [Gl65]. Whether such properties still hold for our sampling projection scheme is not yet known.

Our second objective is to perform a numerical convergence study for several classical non-conservative schemes for two-fluid flows, and compare them to our new scheme. We will observe, surprisingly, that the numerical solutions seem to converge towards the good weak solutions. According to our previous considerations, this behavior is absolutely not obvious. Finally, we compare our new scheme to the GFM in a more complex configuration. We present a hard test case consisting in computing the oscillations of a spherical gas bubble in a compressible liquid. We present the results obtained with the GFM and the random projection scheme.

\section{The two-fluid model}

In this paper, we investigate the numerical approximation of the Euler system for a compressible two-fluid mixture. The density of the mixture is $\rho$, the 
velocity is $u$ and the internal energy is $e$. We denote by $E$ the total energy defined by $E=e+u^{2} / 2$. The pressure is noted $p$. For simplicity, but without loss of generality, we only consider one-dimensional flows. The unknowns depend on the spatial position $x$ and on the time $t$. The PDE system is made of mass, momentum and energy conservation laws

$$
\begin{aligned}
\partial_{t} \rho+\partial_{x}(\rho u) & =0, \\
\partial_{t}(\rho u)+\partial_{x}\left(\rho u^{2}+p\right) & =0, \\
\partial_{t}(\rho E)+\partial_{x}((\rho E+p) u) & =0 .
\end{aligned}
$$

In the case of a one-fluid flow, the pressure would be a function of the density and the internal energy

$$
p=p(\rho, e) .
$$

Because we consider two-fluid flows, our pressure is a function of the density and the internal energy but also of a supplementary unknown $\varphi$, the colour function,

$$
p=p(\rho, e, \varphi) .
$$

The colour function is transported with the flow

$$
\partial_{t} \varphi+u \partial_{x} \varphi=0
$$

Combining this transport equation with the mass conservation law (1) gives a conservative form of the colour function equation

$$
\partial_{t}(\rho \varphi)+\partial_{x}(\rho \varphi u)=0 .
$$

Finally, defining the conservative variables vector

$$
W=(\rho, \rho u, \rho E, \rho \varphi)^{T},
$$

and the flux vector

$$
F(W)=\left(\rho u, \rho u^{2}+p,(\rho E+p) u, \rho \varphi u\right)^{T},
$$

the system (1)-(3), (5), (4) can be written

$$
\partial_{t} W+\partial_{x} F(W)=0 .
$$


For practical computations, we will use the pressure law of a mixture of stiffened gases. We consider a gas and a liquid satisfying stiffened gas laws

$$
p=\left(\gamma_{i}-1\right) \rho e-\gamma_{i} \pi_{i},
$$

with $i=1$ for the gas and $i=2$ for the liquid. The parameters $\gamma_{i}>1$ and $\pi_{i}$ are obtained from physical measurements. The mixture pressure is defined by

$$
p(\rho, e, \varphi)=(\gamma(\varphi)-1) \rho e-\gamma(\varphi) \pi(\varphi) .
$$

The mixture parameters are given by

$$
\begin{aligned}
\frac{1}{\gamma(\varphi)-1} & =\varphi \frac{1}{\gamma_{2}-1}+(1-\varphi) \frac{1}{\gamma_{1}-1} \\
\frac{\gamma(\varphi) \pi(\varphi)}{\gamma(\varphi)-1} & =\varphi \frac{\gamma_{2} \pi_{2}}{\gamma_{2}-1}+(1-\varphi) \frac{\gamma_{1} \pi_{1}}{\gamma_{1}-1}
\end{aligned}
$$

in such a way that $\varphi=1$ in the pure liquid phase and $\varphi=0$ in the pure gas phase. This system has nice mathematical properties: it is hyperbolic and the Riemann problem has a unique solution, even with large data [BHR03].

On the numerical side, the situation is more complicated. For instance, it is well-known that standard conservative finite volume schemes have a poor precision when applied to this kind of flow. Even worse, in some configurations of liquid-gas flows, the explicit Godunov scheme cannot be used because it leads to negative densities.

\section{The Lagrange-projection approach}

For the finite volume approximation, we consider a sequence of times $t_{n}$, $n \in \mathbb{N}$, such that $t_{0}=0$ and $\tau_{n}=t_{n+1}-t_{n}>0$. We also consider mesh points $x_{i+1 / 2}^{n}$ at time $n$. The cell $C_{i}^{n}$ is the interval $] x_{i-1 / 2}^{n}, x_{i+1 / 2}^{n}\left[\right.$. We denote by $x_{i}^{n}$ the center of cell $C_{i}^{n}$

$$
x_{i}^{n}=\frac{x_{i-1 / 2}^{n}+x_{i+1 / 2}^{n}}{2} .
$$

The length of cell $C_{i}^{n}$ is noted $h_{i}^{n}=x_{i+1 / 2}^{n}-x_{i-1 / 2}^{n}$. According to the notations, the mesh is moving but at some time step we will go back to the initial mesh at $n=0$. We note

$$
x_{i}=x_{i}^{0}, \quad C_{i}=C_{i}^{0}, \quad h_{i}=h_{i}^{0}, \quad \text { etc. }
$$


We are looking for an approximation of $W$ in the cell $C_{i}^{n}$

$$
\left.W_{i}^{n} \simeq W(x, t), \quad x \in C_{i}^{n}, \quad t \in\right] t_{n}, t_{n+1}[.
$$

For the numerical approximation, we need an (exact or approximate) Riemann solver. The (exact or approximate) solution of the Riemann problem

$$
\begin{aligned}
\partial_{t} V+\partial_{x} F(V) & =0, \\
V(x, 0) & =\left\{\begin{array}{l}
W_{L} \text { if } x<0, \\
W_{R} \text { if } x>0,
\end{array}\right.
\end{aligned}
$$

is noted

$$
R\left(\frac{x}{t}, W_{L}, W_{R}\right)=V(x, t) .
$$

Each time step of the Lagrange-projection scheme is made of two stages. In the first stage, we approximate the solution with a Lagrange scheme

$$
h_{i}^{n+1 / 2} W_{i}^{n+1 / 2}-h_{i}^{n} W_{i}^{n}+\tau_{n}\left(F_{i+1 / 2}^{n}-F_{i-1 / 2}^{n}\right)=0 .
$$

The Lagrange flux is defined by

$$
\begin{aligned}
F_{i+1 / 2}^{n} & =F\left(W_{i+1 / 2}^{n}\right)-u_{i+1 / 2}^{n} W_{i+1 / 2}^{n}, \\
W_{i+1 / 2}^{n} & =R\left(u_{i+1 / 2}^{n}, W_{i}^{n}, W_{i+1}^{n}\right),
\end{aligned}
$$

where the cell boundary $x_{i+1 / 2}^{n}$ moves at the velocity $u_{i+1 / 2}^{n}$ of the contact discontinuity in the approximation of the Riemann problem between $W_{L}=W_{i}^{n}$ and $W_{R}=W_{i+1}^{n}$

$$
x_{i+1 / 2}^{n+1 / 2}=x_{i+1 / 2}^{n}+\tau_{n} u_{i+1 / 2}^{n} .
$$

In particular, this defines the new size of cell $C_{i}^{n}$

$$
h_{i}^{n+1 / 2}=x_{i+1 / 2}^{n+1 / 2}-x_{i-1 / 2}^{n+1 / 2}=h_{i}^{n}+\tau_{n}\left(u_{i+1 / 2}^{n}-u_{i-1 / 2}^{n}\right) .
$$

This evolution equation is known as the discrete geometric conservation law in the context of finite volume schemes on moving meshes, cf. [FGG01]. In particular, (10) can be generalized to higher dimensions. It permits to avoid the actual computation of the moving mesh.

After the Lagrange stage, we have to go back to the initial Euler mesh. This can be done with several methods 


\subsection{The averaging projection}

In this approach, we average back on the Euler grid, with a simple $L^{2}$ projection

$$
\begin{aligned}
W_{i}^{n+1}= & \frac{\tau_{n}}{h_{i}} \max \left(u_{i-1 / 2}^{n}, 0\right) W_{i-1}^{n+1 / 2}-\frac{\tau_{n}}{h_{i}} \min \left(u_{i+1 / 2}^{n}, 0\right) W_{i+1}^{n+1 / 2} \\
& +\left(1-\frac{\tau_{n}}{h_{i}} \max \left(u_{i-1 / 2}^{n}, 0\right)+\frac{\tau_{n}}{h_{i}} \min \left(u_{i+1 / 2}^{n}, 0\right)\right) W_{i}^{n+1 / 2} .
\end{aligned}
$$

And we go back to the initial Euler grid

$$
C_{i}^{n+1}=C_{i}, \quad h_{i}^{n+1}=h_{i}^{n} .
$$

It can also be written

$$
\begin{array}{r}
W_{i}^{n+1}=W_{i}^{n+1 / 2}-\frac{\tau_{n}}{h_{i}}\left(\max \left(u_{i-1 / 2}^{n}, 0\right)\left(W_{i}^{n+1 / 2}-W_{i-1}^{n+1 / 2}\right)+\right. \\
\left.\min \left(u_{i+1 / 2}^{n+1 / 2}, 0\right)\left(W_{i+1}^{n+1 / 2}-W_{i}^{n+1 / 2}\right)\right) .
\end{array}
$$

In this way, it is clear that the projection step is an upwind approximation of

$$
\partial_{t} W+u \partial_{x} W=0
$$

This method is fully conservative and thus has a bad precision for multi-fluid problems [BHR03]. It is possible to improve the precision by the Saurel-Abgrall approach. It consists in performing a non-conservative projection on the colour function. Instead of projecting $\rho \varphi$ as in (11) we project directly $\varphi$, which gives

$$
\begin{aligned}
\varphi_{i}^{n+1}=\varphi_{i}^{n+1 / 2}-\frac{\tau_{n}}{h_{i}}( & \max \left(u_{i-1 / 2}^{n}, 0\right)\left(\varphi_{i}^{n+1 / 2}-\varphi_{i-1}^{n+1 / 2}\right)+ \\
& \left.\min \left(u_{i+1 / 2}^{n+1 / 2}, 0\right)\left(\varphi_{i+1}^{n+1 / 2}-\varphi_{i}^{n+1 / 2}\right)\right) .
\end{aligned}
$$

This approach results in a globally non-conservative scheme: it induces a numerical mass transfer between the two phases. In the case of the stiffened gas pressure law (9) it can be proved that the resulting scheme preserves constant $(u, p)$ states. 


\subsection{The Glimm projection}

In this approach, we construct a sequence of random or pseudo-random numbers $\omega_{n} \in[0,1]$. According to this number we take

$$
W_{i}^{n+1}=\left\{\begin{array}{ccc}
W_{i-1}^{n+1 / 2} & \text { if } & \omega_{n}<\frac{\tau_{n}}{h_{i}} \max \left(u_{i-1 / 2}^{n}, 0\right), \\
W_{i}^{n+1 / 2} & \text { if } & \frac{\tau_{n}}{h_{i}} \max \left(u_{i-1 / 2}^{n}, 0\right) \leq \omega_{n} \leq 1+\frac{\tau_{n}}{h_{i}} \min \left(u_{i+1 / 2}^{n}, 0\right), \\
W_{i+1}^{n+1 / 2} & \text { if } & \omega_{n}>1+\frac{\tau_{n}}{h_{i}} \min \left(u_{i+1 / 2}^{n}, 0\right) .
\end{array}\right.
$$

And we go back to the initial Euler grid

$$
h_{i}^{n+1}=h_{i}^{n} .
$$

This method is only statistically conservative [Gl65]. It preserves exactly constant velocity-pressure states. The contacts are solved in one point. The drawback is that the solution may be noisy. In particular, for strong shocks the whole method does not converge towards the correct entropy solution.

A good choice for the pseudo-random sequence $\omega_{n}$ is the $\left(k_{1}, k_{2}\right)$ van der Corput sequence, computed by the following $\mathrm{C}$ algorithm

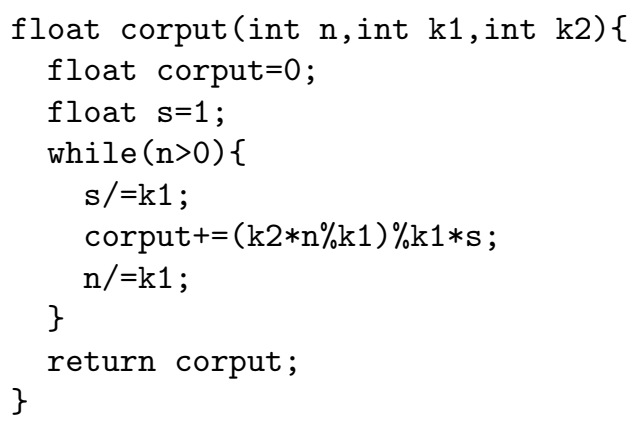

In this algorithm, $k_{1}$ and $k_{2}$ are two numbers that are relatively prime and $k_{1}>k_{2}>0$. For more details, we refer to [Tor99]. In practice, we consider the $(5,3)$ van der Corput sequence.

\subsection{Mixed projection}

In order to improve the convergence of the Glimm approach, it is possible to follow the following mixed projection step. If cell $C_{i}$ and its two neighbours are in the same fluid, i.e., if

$$
\left(\varphi_{i-1}^{n}-\frac{1}{2}\right)\left(\varphi_{i}^{n}-\frac{1}{2}\right)>0 \text { and }\left(\varphi_{i}^{n}-\frac{1}{2}\right)\left(\varphi_{i+1}^{n}-\frac{1}{2}\right)>0,
$$


then, we follow the projection given by (12). In all the other cases, we follow the Glimm projection (13). This approach allows a better precision at the interface because it is resolved in only one point.

\section{The modified ghost fluid approach}

Our ghost fluid method follows the ideas of the ghost fluid method for the poor of Abgrall and Karni [AK01] and its modification by Farhat et al. [FRS08] as well as the ideas of the real ghost fluid method developed by Wang, Liu and Khoo in 2006 [WLK06].

As usual, the interface between the liquid and the gas is identified by a function $\psi$. In fluid $\mathrm{A}$, we have $\psi>0$ and in fluid $\mathrm{B}, \psi<0$. And thus, the interface corresponds to the level set $\psi=0$. As in the previous method, the level set function $\psi$ is transported by the flow

$$
\partial_{t} \psi+u \partial_{x} \psi=0
$$

The pressure law of the liquid and the pressure law of the gas are distinguished by the sign of $\psi$. Obviously, the differences between the colour function model and the level set model are only formal. However, the numerical implementations are rather different, because we separate the discretization of the level set equation (14) and the fluid flow (1-3), respectively, instead of discretizing the coupled system (8) with (6) and (7). In the following the level set

function $\psi$ is approximated in cell $C_{i}$ at time $t_{n}$ by $\psi_{i}^{n}$. For the fluid flow we discretize the evolution equations (1-3) for mass, momentum and energy separately, i.e., in (8), we replace (6) and (7) by the vector of conserved quantities

$$
W=(\rho, \rho u, \rho E)^{T}
$$

and the associated flux vector by

$$
F(W)=\left(\rho u, \rho u^{2}+p,(\rho E+p) u\right)^{T} .
$$


This system is approximated by a finite volume scheme

$$
W_{i}^{n+1}=W_{i}^{n}-\frac{\tau_{n}}{h_{i}}\left(F_{i+1 / 2}^{n,-}-F_{i-1 / 2}^{n,+}\right),
$$

with a possible non-conservative numerical flux, i.e., $F_{i+1 / 2}^{n,-} \neq F_{i+1 / 2}^{n,+}$. In the following we describe in detail how to compute the numerical fluxes. Here we have to distinguish between cells away from the phase interface and cells at the phase interface.

Away from the phase interface:

Let the fluid of two neighbouring cells $C_{i}$ and $C_{i+1}$ belong to the same phase, i.e.,

$$
\psi_{i}^{n} \cdot \psi_{i+1}^{n}>0 .
$$

Then we take the classical conservative Godunov flux

$$
F_{i+1 / 2}^{n,-}=F_{i+1 / 2}^{n,+}=F_{i+1 / 2}^{n}=F\left(R\left(0, W_{i}^{n}, W_{i+1}^{n}\right)\right) .
$$

Thus, only one numerical flux is computed at the cell interface. Note that the spatial order can be improved by using a second-order reconstruction of the primitive variables $\rho, u, p$ and the solution is advanced to the next time step by the finite volume scheme (17). This has been done in [MBKKH09]. In order to be comparable with the Lagrange projection approach, we confine the reconstruction to the first order approximation.

At the phase interface:

Let the phase boundary be lying between cells $C_{i}$ and $C_{i+1}$, where cell $C_{i}$ corresponds to fluid $A$ and cell $C_{i+1}$ to fluid $B$, i.e.,

$$
\psi_{i}^{n} \cdot \psi_{i+1}^{n}<0
$$

Then the left and right state are taken from cells $C_{i+1}$ and $C_{i+2}$, respectively, to ensure access to states of the pure phases

$$
W_{L}=W_{i-1}^{n}, \quad W_{R}=W_{i+2}^{n} .
$$


Solving the Riemann problem between $W_{L}$ and $W_{R}$ we obtain the interfacial velocity $u_{I}$ and the interfacial states to the left and to the right of the material interface $I$

$$
W_{I L}=\lim _{\xi \rightarrow I^{-}} R\left(\xi, W_{L}, W_{R}\right), \quad W_{I R}=\lim _{\xi \rightarrow I^{+}} R\left(\xi, W_{L}, W_{R}\right) .
$$

Here $R\left(\xi, W_{L}, W_{R}\right)$ denotes the self-similar solution of the Riemann problem that is constant along rays $\xi=x / t=$ const. We have thus access to the interfacial states for the densities $\rho_{I L}, \rho_{I R}$ as well as to the interfacial pressure $p_{I}$ and the interfacial velocity $u_{I}$ that are both continuous at the interface. For fluid $A$, the state $\left(\rho_{I L}, u_{I}, p_{I}\right)$ replaces the state of the cell $C_{i}$ and defines the ghost states as in [WLK06]. The numerical fluxes are thus

$F_{i+1 / 2}^{-}=F\left(R\left(0, W_{I L}, W_{I L}\right)\right)=F\left(W_{I L}\right), \quad F_{i+1 / 2}^{+}=F\left(R\left(0, W_{I R}, W_{I R}\right)\right)=F\left(W_{I R}\right)$.

The evolution equation (17) is slightly modified by

$$
W_{i}^{n+1}=W_{I L}-\frac{\tau_{n}}{h_{i}}\left(F_{i+1 / 2}^{n,-}-F_{i-1 / 2}^{n,+}\right)
$$

and

$$
W_{i+1}^{n+1}=W_{I R}-\frac{\tau_{n}}{h_{i+1}}\left(F_{i+3 / 2}^{n,-}-F_{i+1 / 2}^{n,+}\right)
$$

[Figure 1 about here.]

This procedure is sketched in Fig. 1. As a consequence, only single-phase Riemann problems are solved for each cell interface of fluid $A$ to provide the numerical fluxes with the ghost cells as boundary conditions at the phase boundary. Then the same procedure is used for fluid $B$. Thus near the phase boundary, two fluxes $\mathbf{F}_{i+\frac{1}{2}}^{n, \pm}$, one for each fluid, are defined.

On the other hand, the level set function has also to be advanced. This is done first by solving numerically (14) with a standard upwind non-conservative finite volume scheme

$$
\psi_{i}^{n+1,-}=\psi_{i}^{n}-\frac{\tau_{n}}{h_{i}}\left(\max \left(u_{i-1 / 2}^{n}, 0\right)\left(\psi_{i}^{n}-\psi_{i-1}^{n}\right)+\min \left(u_{i+1 / 2}^{n}, 0\right)\left(\psi_{i+1}^{n}-\psi_{i}^{n}\right)\right)
$$


Periodically, the approximation of the level set function is reinitialized in such a way that it remains a signed distance to the interface. This is formally obtained through the numerical solution of a Hamilton-Jacobi equation

$$
\begin{aligned}
\partial_{\tau} \widetilde{\psi}(x, \tau)+a(\widetilde{\psi}) \partial_{x} \widetilde{\psi} & =S(\widetilde{\psi}) \\
\widetilde{\psi}(x, \tau=0) & =\psi_{i}^{n+1,-}, \quad x \in C_{i},
\end{aligned}
$$

with

$$
a(\widetilde{\psi})=S(\widetilde{\psi}) \frac{\partial_{x} \widetilde{\psi}}{\left|\partial_{x} \widetilde{\psi}\right|}, \quad S(\widetilde{\psi})=\left\{\begin{array}{ccc}
-1 & \text { if } & \widetilde{\psi}<0, \\
0 & \text { if } & \widetilde{\psi}=0, \\
1 & \text { if } & \widetilde{\psi}>0,
\end{array} .\right.
$$

The level set function is replaced by the reinitialized level set function, i.e., we take

$$
\psi_{i}^{n+1}=\widetilde{\psi}(x, \tau=\infty), \quad x \in C_{i} .
$$

This procedure is described in more details in [MBKKH09].

Finally, due to the update of the level set function, the phase in a cell may change from one fluid to the other. This situation corresponds to a change of the sign between time step $n$ and time $n+1$, i.e., when $\psi_{i}^{n} \cdot \psi_{i}^{n+1}<0$. In this case, it is necessary to also update $W_{i}^{n+1}$ in the corresponding cell because the state corresponds to the wrong equation of state. The fluid variables are recalculated using the equation of state of the new fluid. The cell being very close to the phase boundary, the velocities and the pressure, which are constant for both fluids at the phase boundary, are preserved, see Eq. (19)

$$
\begin{aligned}
W_{i}^{n+1} & =\rho_{i}^{n+1}\left(1, u_{i}^{n+1}, E_{i}^{n+1}\right)^{T} \\
& \rightarrow p\left(\rho_{i}^{n+1}, e_{i}^{n+1}, \psi_{i}^{n}\right) \rightarrow \tilde{e}\left(\rho_{i}^{n+1}, p_{i}^{n+1}, \psi_{i}^{n+1}\right) \rightarrow \\
W_{i}^{n+1} & =\rho_{i}^{n+1}\left(1, u_{i}^{n+1}, \tilde{E}_{i}^{n+1}\right) .
\end{aligned}
$$

This modification was suggested by Barberon [BA02]. In addition to this approach, we propose to modify the density as well, because there will be large 
jumps in the density for two-phase fluids such as water and air. Also the density is simply replaced by the density of the corresponding ghost cell. More precisely, if $\psi_{i}^{n} \cdot \psi_{i}^{n+1}<0$, and if $\psi_{i}^{n} \cdot \psi_{i+1}^{n}<0$, then, before computing the next time-step, we substitute the density $\rho_{i}^{n+1}$ in (19) by

$$
\rho_{i}^{n+1} \leftarrow\left\{\begin{array}{ll}
\rho_{I R}, & \text { if cell } i \text { goes to fluid B } \\
\rho_{I L}, & \text { if cell } i \text { goes to fluid A }
\end{array}\right. \text {. }
$$

This construction implies that the whole resulting scheme will preserve constant $(u, p)$ states. On the other hand, it is obvious that the scheme is not conservative. For instance, (20) implies a mass and an energy transfer between the two fluids. This modification of the density (20) is similar to [FRS08], where

the solution of the finite volume scheme in primitive variable $W_{i}^{n+1}$ is substituted by the solution of the Riemann problem corresponding to the new fluid, i.e.,

$$
W_{i}^{n+1}=W_{I *},
$$

where ${ }^{*}$ is either the left or the right state. This procedure ensures that the velocity and the pressure are continuous at a contact discontinuity.

\section{Numerical results}

\subsection{Introduction}

In this part, we compare the three Lagrange-projection approaches (averaging projection, Glimm projection and mixed projection) and the two Eulerian methods (Saurel-Abgrall and ghost fluid method). Before presenting the results, we give some information on the discretization used for these algorithms.

For the Lagrange-projection plan, we use a uniform mesh because it allows to perform the computations on a GPU.

For the Eulerian approach, we apply multi-scale grid adaptation [MÜ03]. In fact, the problem is the precision of the interface position, that can be improved by using more cells at the phase interface than elsewhere. The coarse discretization consists of 100 cells to which the multi-scale transformation is applied, see [MBKKH09]. We denote by $L$ the number of refinement levels, i.e., 
the uniform grid on the finest level consists of $2^{L} * 100$ cells. The threshold value in the grid adaptation is chosen as $\epsilon=10^{-5}$ such that the error obtained with the multiscale grid adaptation is comparable with the error obtained with a uniform grid. Tests are performed with a CFL number of 0.7 .

\subsection{Academic validation}

The first test consists in a two-fluid shock tube problem. The stiffened gas parameters are given in Table 1.

\section{[Table 1 about here.]}

The interface between the two fluids is located at time $t_{0}=0 \mathrm{~s}$ at position $x=0 \mathrm{~m}$. The computational domain is $[-1 ; 1] \mathrm{m}$ and the final time is $t_{A}=0.5 \mathrm{~s}$. Left and right initial states are given in Table 2.

[Table 2 about here.]

The Lagrange-projection approach, the non-conservative projection, the Glimm projection and the mixed projection are compared. We observe numerical convergence in the $L^{1}$-norm for the three methods and that Glimm and mixed projection are more precise than the averaging projection, see Figure 2. The convergence rate for the averaging projection is approximately 0.5 while it is approximately 0.8 for the two others.

For the Eulerian approach, the non-conservative methods of Saurel-Abgrall and the GFM are compared. Obviously the GFM is more precise, see Figure 2. The convergence rate is approximately 0.5 for the Saurel-Abgrall approach and 0.6 for the GFM.

[Figure 2 about here.]

\section{3. $1 D$ academic shock-interface interaction}

The interface between the two fluids is located at time $t_{0}=0 \mathrm{~s}$ at position $x_{I 0}=1 \mathrm{~m}$. The two fluids are moving to the left at velocity $u=-1 \mathrm{~m} / \mathrm{s}$. Fluid (A) is on the left, while fluid (B) is on the right. A shock is arriving from

position $x_{S A}=-4 \mathrm{~m}$ at velocity $\sigma=4 \mathrm{~m} / \mathrm{s}$. The initial position of the interface 
and the shock are chosen in such way that they will interact at position $x_{S}=0$ $\mathrm{m}$ at time $t_{I}=1 \mathrm{~s}$. The computational domain is $[-5 ; 2] \mathrm{m}$. The material parameters are listed in Table 4.

When the shock wave and the interface have interacted at time $t_{I}=1 \mathrm{~s}$, the solution is simply given by the solution of a two-fluid Riemann problem between states (A) and (B). In fact, the exact solution is the exact solution of the Riemann problem between the states $W_{A}$ and $W_{B}$ at final time $t=t_{A}-t_{I}$.

At time $t_{A}=1.5 \mathrm{~s}$, the shock in the fluid (B) is located at position $x_{S B}=$ $1.56 \mathrm{~m}$, the interface is located at position $x_{I 1}=0.93 \mathrm{~m}$ and the rarefaction wave in the fluid (A) is between positions $x_{R A L}=-1.55 \mathrm{~m}$ and $x_{R A R}=-0.92$ $\mathrm{m}$. The solution structure is sketched in Figure 3. The numerical data are recorded in Table 3.

[Table 3 about here.]

[Table 4 about here.]

[Figure 3 about here.]

\subsubsection{Lagrange plus projection schemes}

In this case, we observe that the Glimm approach does not converge. This behavior depends on the strength of the shock wave. A typical plot is given in Figure 4, where we compare the exact and the approximated densities at time $t_{A}=1.5 \mathrm{~s}$.

[Figure 4 about here.]

We also provide in Figure 5 a comparison of the mixed and averaging projection schemes for the densities, where we discretize the interval $[-5 ; 2] \mathrm{m}$ by 500 cells. The CFL number is fixed to 0.7. It is interesting to observe that the interface position is very well resolved (in only one mesh point) by the mixed projection scheme and that this good resolution of the contact wave also implies an improvement of the precision in the left rarefaction wave.

[Figure 5 about here.] 


\subsubsection{Modified ghost fluid method}

In order to compare the non-conservative methods of Saurel-Abgrall and the GFM, several numerical solutions are compared with the exact solution.

In Figure 6 the density profiles are shown at $t=1.5 \mathrm{~s}$ in case of $L=10$. This corresponds to a cell length of $68 \mu \mathrm{m}$. Both methods fit very well with the exact solution. That means that the interface, the shock and the rarefaction waves are well resolved.

[Figure 6 about here.]

\subsubsection{Convergence study}

We compare the numerical convergence in the $L^{1}$ norm for the three Lagrangianprojection approaches and for the two Eulerian approaches in Figure 7. For the Eulerian methods the convergence study is performed for grids having 5 to 13 refinement levels $L$. We note in Figure 4 that the Glimm approach does not converge but the four other procedures have small convergence errors. The convergence rate for the averaging projection and for the non-conservative method of Saurel-Abgrall is approximately 0.5 , while it is 0.8 for the mixed projection and the GFM.

In fact, the Glimm approach does not converge in all cases, we have to use the mixed projection to avoid oscillations. The best error convergence is achieved with mixed-projection and the GFM.

[Figure 7 about here.]

\subsection{Air-water test case}

The second test case is a shock of Mach number 0.67 starting at position $x_{S A}=-3 \mathrm{~m}$ running at velocity $\sigma=3000 \mathrm{~m} / \mathrm{s}$ in the liquid towards an interface located at position $x_{I 0}=0.1 \mathrm{~m}$. It interacts with air at time $t_{I}=1 \mathrm{~ms}$ at position $x_{s}=0 \mathrm{~m}$. The water ahead of the shock and the air are moving towards the shock at velocity of $100 \mathrm{~m} / \mathrm{s}$. The computational domain is $[-4 ; 2]$ $\mathrm{m}$. 
The exact solution is the exact solution of the Riemann problem between the states $U_{A}$ and $U_{B}$ at final time $t=t_{A}-t_{I}$.

At time $t_{A}=1.5 \mathrm{~ms}$, the shock in the fluid (B) is located at position $x_{S B}=$ $1.45 \mathrm{~m}$, the interface is located at position $x_{I 1}=1.18 \mathrm{~m}$ and the rarefaction wave in the fluid (A) is between positions $x_{R A L}=-0.88 \mathrm{~m}$ and $x_{R A R}=0.4 \mathrm{~m}$. The configuration is sketched in Figure 3 and the different intermediate states are summarized in Table 5. The material parameters for the fluids are listed in Table 6 .

[Table 5 about here.]

[Table 6 about here.]

\subsubsection{Density graph}

In Figure 8, we compare the density obtained with the two Eulerian approaches and the one obtained with the mixed projection to the exact solution at time $t_{A}=1.5 \mathrm{~ms}$. For the Eulerian approaches, we use a grid with $L=7$ refinement levels that corresponds to a finest cell length of $470 \mu \mathrm{m}$ for a uniform grid. The global result is presented in the middle as well as a zoom of the shock position (bottom left), a zoom of the plateau between the rarefaction wave and the interface (top left) and a zoom very close to the contact position (right). In the latter one, cell centers are marked by diamonds for the numerical results.

The Saurel-Abgrall approach generates oscillations at the right kink of the rarefaction wave due to the interaction between the shock and the interface. This is not the case for the GFM and the mixed projection which match quite well with the exact solution.

At the interface, there is a smearing of the density for the Saurel-Abgrall approach. This can be seen in the zoom on the right, where the density decreases slowly instead of jumping as in case of the GFM or the mixed projection. Due to the construction of the ghost fluid method, we obtain the desired jump at the interface but this jump is a little bit shifted compared to the exact solution. As the cell centers are represented by diamonds in the figure, for $L=7$ refinement 
levels, we can see a shift in the position of the interface by 2 cells. With the mixed projection, there is no smearing on the interface position but the jump is a little bit shifted too.

Concerning the shock position which is only visible in the zoom due to the big jump of the density, we notice that its position is well predicted with the GFM and the mixed projection. This is different for the Saurel-Abgrall approach.

[Figure 8 about here.]

\subsubsection{Saurel-Abgrall approximation}

As we saw in Section 4.4.1, the Saurel-Abgrall method is not really consistent with the exact solution. To see if the problem comes from a lack of resolution, we plot the density for the two Eulerian approaches with a very fine grid corresponding to $L=12$ refinement levels in Figure 9. We note that

(i) in the rarefaction wave, the amplitude and the number of oscillations are largely reduced;

(ii) as the cell centers are represented by diamonds, for $L=12$ refinement levels, we can see a shift in the position of the interface about 4 cells;

(iii) concerning the shock position, the shift reduces as much as the smearing region at the interface becomes smaller with grid refinement.

[Figure 9 about here.]

From these observations we conclude that the oscillations and the error in the shock position for the S-A approach are only caused by the previous shockinterface interaction. To verify this we perform a computation that starts at $t_{I}=1 \mathrm{~ms}$, i.e., a two-phase Riemann problem is solved. Then the results are in good agreement with the exact solution everywhere. This result is shown in Figure 10 in comparison with the exact solution and the numerical solution with oscillations.

[Figure 10 about here.] 


\subsubsection{Convergence Study}

The $L^{1}$-error of the density is summarized in Figure 11. The order of convergence for the Saurel-Abgrall approach is 0.5. Concerning the GFM, the error seems to tend to the same order under grid refinement, see Figure 11. For the mixed projection, the convergence rate is approximately 0.8 .

[Figure 11 about here.]

\subsubsection{Error at the interface}

In this section the error in the interface position is discussed in more detail. For the Saurel-Abgrall approach, a gas mass fraction of 0.5 is chosen to characterize the interface position. For the GFM, it corresponds to the zero level set. As there is no smearing of the interface with the mixed projection method, the interface position corresponds to the jump from 1 to 0 in the gas mass fraction. The error of this position and the exact position of the interface is computed and used to plot Figure 12, where we represent the logarithm of the difference between the exact position and the numerical position of the interface.

The Saurel-Abgrall approach shows a convergence rate of 0.5. For the GFM, the error decreases under grid refinement. The order might be less than 0.5 with several additional refinement levels. However, the interface position is always shifted compared to the exact solution although the error reduces under grid refinement. This is due to the non-conservative discretization near to the phase boundary, where two fluxes are computed at a cell interface. The mixed projection gives a convergence rate of 0.65 .

In Figure 12, we show a graph of the error between the exact position and the numerical position of the interface.

[Figure 12 about here.]

\section{Gas bubble oscillations}

\subsection{Introduction}

In this section we apply the random projection scheme to the bubble oscillation test case described in [HMM08, MBKKH09, Ma10]. Despite the quasi 
one-dimensional framework, the test case implies long computations and fine meshes. We compare with the results obtained with the GFM on locally refined grids.

\subsubsection{Model}

To study the spherical bubble of gas in the liquid phase we consider the $3 \mathrm{D}$-Euler equations. We assume that the flow is invariant under rotation. We note $x$ the space variable along the radius of the bubble and $t$ the time, see Figure 13.

[Figure 13 about here.]

With the spherical symmetry the unknowns are the density $\rho$, the radial velocity $u$, the internal energy $e$ and the fraction mass of gas $\varphi$ and they depend only on $(x, t)$. We can write the 3D-Euler equations in the following form:

$$
\begin{aligned}
\partial_{t}(A \rho)+\partial_{x}(A \rho u) & =0, \\
\partial_{t}(A \rho u)+\partial_{x}\left(A\left(\rho u^{2}+p\right)\right) & =p A^{\prime}(x), \\
\partial_{t}(A \rho E)+\partial_{x}(A(\rho E+p) u) & =0, \\
\partial_{t}(A \rho \varphi)+\partial_{x}(A \rho \varphi u) & =0,
\end{aligned}
$$

where $A(x)=x^{2}$ appears because of the symmetry. We recall that $p$ satisfies a mixture stiffened gas pressure law defined by (9) with different material parameters for the gas and the liquid.

We write the system (21)-(22) in the condensed form:

$$
\partial_{t}(A W)+\partial_{x}\left(A F_{1}(W)\right)=A^{\prime}(x) B(W)
$$

where $W=(\rho, \rho u, \rho E, \rho \varphi)^{T}, F_{1}(W)=\left(\rho u, \rho u^{2}+p,(\rho E+p) u, \rho \varphi u\right)^{T}$, and the source term is

$$
A^{\prime}(x) B(W)=A^{\prime}(x)(0, p, 0,0)^{T} .
$$




\subsubsection{Boundary conditions and initial data}

We assume that we have a bubble of gas in a liquid phase. Then the initial conditions are of the form

$$
Y(x, 0)=\left\{\begin{array}{l}
Y_{L}, \text { if } x<R_{b} \\
Y_{R}, \text { if } x>R_{b}
\end{array},\right.
$$

where $R_{b}$ is the initial radius of the bubble and $Y=(\rho, u, p, \varphi)^{T}$.

For the boundary conditions, we take wall boundary condition on the right side and on the left side.

The initial left and right states are computed as it was down in [MBKKH09], we briefly recall this method. The equilibrium radius $R_{e q}$ is the radius, where the pressure in the bubble is equal to the pressure acting from outside, there is a static equilibrium. Hence

$$
p_{i}\left(R_{e q}\right)=p_{0}+\frac{2 \sigma}{R_{e q}},
$$

with $p_{0}$ being the ambient pressure and $\sigma$ the surface tension. Neglecting surface tension we have:

$$
p_{i}\left(R_{e q}\right)=p_{0}
$$

With the ideal gas law $p V=n R T$ we get the number of molecules (in [mol]) inside the bubble ( $R$ is the specific gas constant):

$$
n=\frac{p_{0} V_{0}}{T_{0} R}=\frac{p_{0} 4 \pi R_{e q}^{3}}{3 T_{0} R},
$$

where $T_{0}$ is the ambient temperature. If we now choose an arbitrary radius, e.g., the starting radius, we use the adiabatic gas law to get the pressure inside the bubble

$$
p_{i}\left(R_{b}\right)=p_{0}\left(\frac{R_{e q}^{3}}{R_{b}^{3}}\right)^{\gamma_{g}},
$$

where $\gamma_{g}$ is the polytropic exponent of the gas. Once we have the pressure $p_{i}\left(R_{b}\right)$, then with the ideal gas law we know the temperature

$$
T\left(R_{b}\right)=T_{0}\left(\frac{R_{e q}}{R_{b}}\right)^{3\left(\gamma_{g}-1\right)} .
$$


As $p \rho^{-\gamma_{g}}=$ const, we obtain

$$
\rho\left(R_{b}\right)=\rho_{0, g a s}\left(\frac{p_{i}\left(R_{b}\right)}{p_{0}}\right)^{\frac{1}{\gamma_{g}}}
$$

where $\rho_{0, \text { gas }}$ is the density of the gas at $\left(p_{0}, T_{0}\right)$.

We assume that $T_{0}=293.15 \mathrm{~K}, p_{0}=10^{5} \mathrm{~Pa}, \gamma_{g}=1.4, \rho_{0, \text { gas }}=1.204 \mathrm{~kg} \cdot \mathrm{m}^{-3}$, $\gamma_{l i q}=3$ and $\rho_{0, l i q}=1000 \mathrm{~kg} \cdot \mathrm{m}^{-3}$. We study two different test cases where the difference are the initial radius of the bubble and the speed of sound in the liquid phase. The first case that is easier will be called the "weakly compressed bubble" and the second test case will be called the "strongly compressed bubble".

The weakly compressed bubble: We study the behavior of a spherical bubble that is slightly dilated. We assume that the maximal radius of the bubble is $R_{\max }=R_{b}=0.7469 \times 10^{-4} \mathrm{~m}$ with an equilibrium radius of $R_{e q}=0.692 \times$ $10^{-4} \mathrm{~m}$, cf. [MBKKH09]. We then obtain:

$$
\begin{aligned}
p\left(R_{b}\right) & =72567.68471 \mathrm{~Pa}, \\
T\left(R_{b}\right) & =267.4867417 \mathrm{~K}, \\
\rho\left(R_{b}\right) & =0.9575410293 \mathrm{~kg} \cdot \mathrm{m}^{-3} .
\end{aligned}
$$

To obtain slowly oscillations we assume a speed of sound in the liquid of $c_{0, l i q}=$ $50 \mathrm{~m} . \mathrm{s}^{-1}$. Using the relation

$$
\pi_{l i q}=\frac{c_{0, l i q}^{2} \times \rho_{0, l i q}}{\gamma_{l i q}}-p_{0},
$$

we obtain $\pi_{l i q}=7.33333333 \times 10^{5} \mathrm{~Pa}$.

The strongly compressed bubble: We study a cavitation bubble inducted by a laser pulse. From experiments performed at the University of Göttingen, cf. [MBKKH09], we are given the maximal radius of the bubble $R_{\max }=R_{b}=$ $0.7469 \times 10^{-3} \mathrm{~m}$. Thus the equilibrium radius is approximately $R_{e q}=0.692 \times$ $10^{-4} \mathrm{~m}$, cf. [MBKKH09]. 
We then obtain:

$$
\begin{aligned}
p\left(R_{b}\right) & =4.578711364 \mathrm{~Pa}, \\
T\left(R_{b}\right) & =16.87727242 \mathrm{~K}, \\
\rho\left(R_{b}\right) & =0.0009575410292 \mathrm{~kg} \cdot \mathrm{m}^{-3} .
\end{aligned}
$$

We assume a speed of sound in the liquid of $c_{0, l i q}=1500 \mathrm{~m} \cdot \mathrm{s}^{-1}$. Using the relation (28) we obtain $\pi_{l i q}=7.49900000 e 8 \mathrm{~Pa}$.

\subsection{Numerical scheme with Glimm procedure}

In order to solve the system (23), we decide to apply a Lagrangian approach as it was done in the previous part. The motivation for this choice is that the Eulerian approach is known to have poor precision and to smear the interface. Note that for the Lagrangian approaches to be presented below the approximated $\varphi$ takes only two values: 1 in the gas and 0 in the liquid.

As it is explain in Section 2, we look for an approximation

$$
W_{i}^{n}=\frac{1}{V_{i}^{n}} \int_{x_{i-1 / 2}^{n}}^{x_{i+1 / 2}^{n}} A(x) W\left(x, t^{n}\right) d x,
$$

where

$$
V_{i}^{n}=\int_{x_{i-1 / 2}^{n}}^{x_{i+1 / 2}^{n}} A(x) d x
$$

is the volum of the cell $\left.C_{i}^{n}=\right] x_{i-1 / 2}^{n} ; x_{i+1 / 2}^{n}\left[\right.$. The boundaries of the cell $C_{i}^{n}$ are moving from $t_{n}$ to $t_{n+1}$ and we use the same notation as in Section 2. The bubble interface is supposed to coincide with a cell edge at time $t=0 \mathrm{~s}$.

To write the two numerical schemes, we have to define:

$$
\begin{aligned}
A_{i+1 / 2}^{n} & =A\left(x_{i+1 / 2}^{n}\right)=\left(x_{i+1 / 2}^{n}\right)^{2}, \\
A_{i+1 / 2}^{n+1 / 4} & =A\left(x_{i+1 / 2}^{n}+u_{i+1 / 2}^{n} \frac{\tau_{n}}{2}\right)=\left(x_{i+1 / 2}^{n}+u_{i+1 / 2}^{n} \frac{\tau_{n}}{2}\right)^{2}, \\
A_{i}^{n+1 / 4} & =A\left(x_{i+1 / 2}^{n}+u_{i+1 / 2}^{n} \frac{\tau_{n}}{2}+x_{i-1 / 2}^{n}+u_{i-1 / 2}^{n} \frac{\tau_{n}}{2}\right), \\
& =\left(x_{i+1 / 2}^{n}+u_{i+1 / 2}^{n} \frac{\tau_{n}}{2}+x_{i-1 / 2}^{n}+u_{i-1 / 2}^{n} \frac{\tau_{n}}{2}\right)^{2}, \\
V_{i}^{n+1 / 2} & =\int_{x_{i-1 / 2}^{n+1 / 2}}^{x_{i+1 / 2}^{n+1 / 2}} A(x) d x,
\end{aligned}
$$




\subsubsection{First algorithm.}

To obtain an explicit first order finite volume scheme, we use a splitting method to solve the system (23):

- we use a Lagrange-projection scheme to solve

$$
\partial_{t}(A W)+\partial_{x}\left(A F_{1}(W)\right)=0,
$$

between time $t_{n}$ and $t_{n+1}$ with the initial condition

$$
\left.W^{n}(x)=W_{i}^{n} \text { if } x \in\right] x_{i-\frac{1}{2}}^{n} ; x_{i+\frac{1}{2}}^{n}[.
$$

The solution of this step is denoted by $W^{n+\frac{1}{2}}$.

- we solve

$$
\partial_{t}(A W)=A^{\prime}(x) B(W),
$$

between time $t_{n}$ and $t_{n+1}$ with the initial condition

$$
\left.W(x)=W_{i}^{n+\frac{1}{2}} \text { if } x \in\right] x_{i-\frac{1}{2}}^{n} ; x_{i+\frac{1}{2}}^{n}[.
$$

We explain the different steps of the algorithm to obtain $W^{n+1}$ with $W^{n}$ :

Lagrange step: We integrate the system (36) on the space-time trapezoid

$$
\begin{aligned}
Q=\{(x, t) & \in \mathbb{R}^{2} \mid t_{n}<t<t_{n+1}, x_{i-\frac{1}{2}}^{n}+t \times v_{i-1 / 2}^{n}<x<x_{i+\frac{1}{2}}^{n}+t \times v_{i+1 / 2}^{n}(\{0) \\
& \left.=\bigcup_{t_{n}<t<t_{n+1}}\right] x_{i-\frac{1}{2}}^{n}+t \times v_{i-1 / 2}^{n} ; x_{i+\frac{1}{2}}^{n}+t \times v_{i+1 / 2}^{n}[\times\{t\}
\end{aligned}
$$

Using the Green's formula, we obtain the following approximation:

$$
\begin{aligned}
& \int_{Q} \partial_{t}(A W)+\partial_{x}\left(A F_{1}(W)\right) d x d t=0 \\
\Rightarrow & V_{i}^{n+1 / 2} W_{i}^{n+\frac{1}{2},-}=V_{i}^{n} W_{i}^{n}-\tau_{n}\left(A_{i+1 / 2}^{n} F_{1, i+1 / 2}^{n}-A_{i-1 / 2}^{n} F_{1, i-1 / 2}^{n}\right),(
\end{aligned}
$$

where $F_{1, i+1 / 2}^{n}$ is the Lagrangian flux:

$$
\begin{aligned}
F_{1, i+1 / 2}^{n} & =F\left(W\left(u_{i+1 / 2}^{n}, W_{i}^{n}, W_{i+1}^{n}\right)\right)-u_{i+1 / 2}^{n} W\left(u_{i+1 / 2}^{n}, W_{i}^{n}, W_{i+1}^{n}\right), \\
& =\left(0, p_{i+1 / 2}^{n}, u_{i+1 / 2}^{n} p_{i+1 / 2}^{n}, 0\right)^{T},
\end{aligned}
$$


and $W\left(u_{i+1 / 2}^{n}, W_{i}^{n}, W_{i+1}^{n}\right)$ is obtained with an exact solution of the Riemann problem:

$$
\begin{gathered}
\partial_{t} W+\partial_{x} F_{1}(W)=0 \\
W(x, 0)=\left\{\begin{array}{l}
W_{i}^{n}, \text { if } x<0 \\
W_{i+1}^{n}, \text { if } x>0
\end{array}\right.
\end{gathered}
$$

on the ray $\frac{x}{t}=u_{i+1 / 2}^{n}$. As $A$ is a smooth function, we assume that $A$ is constant in a neighborhood of $x_{i+1 / 2}^{n}$ and we can write system (23) in the form (44) in the neighborhood of $x_{i+1 / 2}^{n}$.

Projection step: With the previous part we are able to compute $W_{i}^{n+\frac{1}{2},-}$ (see (43)) which is an approximation of $W\left(x, t_{n+1}\right)$ on cell $\left.C_{i}^{n+1 / 2}=\right] x_{i-\frac{1}{2}}^{n+1 / 2} ; x_{i+\frac{1}{2}}^{n+1 / 2}[$. To go back to the original grid we use the mixed projection but we do not write it similarly as in Section 2.3, because we need to take into account $A$. In fact we have to distinguish two cases:

- if we are not at the interface, it means that $\left(\varphi_{i-1}^{n}-\frac{1}{2}\right)\left(\varphi_{i}^{n}-\frac{1}{2}\right)>0$ and $\left(\varphi_{i}^{n}-\frac{1}{2}\right)\left(\varphi_{i+1}^{n}-\frac{1}{2}\right)>0$, we use the averaging projection which takes the form:

$$
\begin{aligned}
& W_{i}^{n+\frac{1}{2}}=\frac{1}{V_{i}^{n}} \int_{x_{i-\frac{1}{2}}^{n}}^{x_{i+\frac{1}{2}}^{n}} A(x) W^{n+\frac{1}{2},-}(x) d x \\
& =\frac{1}{V_{i}^{n}} \int_{x_{i-\frac{1}{2}}^{n}}^{x_{i-\frac{1}{2}}^{n}+\max \left(0, u_{i-\frac{1}{2}}^{n}\right) \tau_{n}} A(x) W^{n+\frac{1}{2},-}(x) d x \\
& +\frac{1}{V_{i}^{n}} \int_{x_{i-\frac{1}{2}}^{n}}^{x_{i+\frac{1}{2}}^{n}+\max \left(0, u_{i-\frac{1}{2}}^{n}\right) \tau_{n}} A(x) W^{n+\frac{1}{2},-}(x) d x \\
& +\frac{1}{V_{i}^{n}} \int_{x_{i-\frac{1}{2}}^{n}+\min \left(0, u_{i+\frac{1}{2}}^{n}\right) \tau_{n}}^{x_{i+\frac{1}{2}}^{n}} A(x) W^{n+\frac{1}{2},-}(x) d x \\
& =\frac{V_{i, L}^{n+1 / 2}}{V_{i}^{n}} W_{i-1}^{n+\frac{1}{2},-}+\frac{V_{i, M}^{n+1 / 2}}{V_{i}^{n}} W_{i}^{n+\frac{1}{2},-}+\frac{V_{i, R}^{n+1 / 2}}{V_{i}^{n}} W_{i+1}^{n+\frac{1}{2},-}
\end{aligned}
$$


where

$$
\begin{aligned}
V_{i, L}^{n+1 / 2} & =\int_{x_{i-\frac{1}{2}}^{n}}^{x_{i-\frac{1}{2}}^{n}+\max \left(0, u_{i-\frac{1}{2}}^{n}\right) \tau_{n}} A(x) d x, \\
V_{i, M}^{n+1 / 2} & =\int_{x_{i-\frac{1}{2}}^{n}+\max \left(0, u_{i-\frac{1}{2}}^{n}\right) \tau_{n}}^{x_{i+\frac{1}{2}}^{n}+\min \left(0, u_{i+\frac{1}{2}}^{n}\right) \tau_{n}} A(x) d x, \\
V_{i, R}^{n+1 / 2} & =\int_{x_{i-\frac{1}{2}}^{n}+\min \left(0, u_{i+\frac{1}{2}}^{n}\right) \tau_{n}}^{x_{i+\frac{1}{2}}^{n}} A(x) d x .
\end{aligned}
$$

- if we are at the interface, we use the Glimm projection. We construct a sequence of random or pseudo-random numbers $\omega_{n} \in[0,1]$. According to this number we take

$$
W_{i}^{n+\frac{1}{2}}=\left\{\begin{array}{ccc}
W_{i-1}^{n+\frac{1}{2},-} & \text { if } & \omega_{n} V_{i}^{n}<V_{i, L}^{n+1 / 2}, \\
W_{i}^{n+\frac{1}{2},-} & \text { if } & V_{i, L}^{n+1 / 2} \leq \omega_{n} V_{i}^{n} \leq V_{i}^{n}-V_{i, R}^{n+1 / 2}, \\
W_{i+1}^{n+\frac{1}{2},-} & \text { if } & \omega_{n} V_{i}^{n}>V_{i}^{n}-V_{i, R}^{n+1 / 2} .
\end{array}\right.
$$

Source term step: Now, we have to solve (38) with the initial condition (39). To obtain an explicit first order finite volume scheme, we integrate the system (38) on the space-time trapezoid

$$
\left.Q^{\prime}=\right] x_{i-\frac{1}{2}}^{n} ; x_{i+\frac{1}{2}}^{n}[\times] t_{n} ; t_{n+1}[.
$$

Thus, we obtain for the time derivation

$$
\int_{Q^{\prime}} \partial_{t}(A W) d x d t=V_{i}^{n} W_{i}^{n+1}-V_{i}^{n} W_{i}^{n+\frac{1}{2}}
$$

and we apply for the integration of the source term a first order approximation

$$
\int_{Q^{\prime}} A^{\prime}(x) B(W) d x d t=\tau_{n} B\left(W_{i}^{n+\frac{1}{2}}\right)\left(A_{i+1 / 2}^{n}-A_{i-1 / 2}^{n}\right),
$$

resulting in the following formula

$$
V_{i}^{n} W_{i}^{n+1}=V_{i}^{n} W_{i}^{n+\frac{1}{2}}+\tau_{n} B\left(W_{i}^{n+\frac{1}{2}}\right)\left(A_{i+1 / 2}^{n}-A_{i-1 / 2}^{n}\right) .
$$




\subsubsection{Second algorithm.}

For smooth solutions we can write the system (21)-(22) in the form:

$$
\begin{aligned}
\partial_{t}(A \rho)+\partial_{x}(A \rho u) & =0 \\
\partial_{t}(A \rho u)+\partial_{x}\left(A \rho u^{2}\right)+A \partial_{x} p & =0 \\
\partial_{t}(A \rho E)+\partial_{x}(A(\rho E+p) u) & =0, \\
\partial_{t}(A \rho \varphi)+\partial_{x}(A \rho \varphi u) & =0,
\end{aligned}
$$

where $A=A(x)=x^{2}$ is a smooth function. We write the system (52)-(53) in the condensed form:

$$
\partial_{t}(A W)+\partial_{x}\left(A F_{2}(W)\right)+A(x) \partial_{x} G(W)=0,
$$

where $W=(\rho, \rho u, \rho E, \rho \varphi)^{T}, F_{2}(W)=\left(\rho u, \rho u^{2},(\rho E+p) u, \rho \varphi u\right)^{T}$, and the source term is

$$
A(x) \partial_{x} G(W)=A(x)\left(0, \partial_{x} p, 0,0\right)^{T} .
$$

Remark 1. The second component of the flux $F_{2}$ is different to the second component of the flux $F_{1}$ of the first model.

In order to obtain a time-centered finite difference scheme, we adapt the method proposed in [BAB86] and we write:

$$
\begin{aligned}
& V_{i}^{n+1 / 2} W_{i}^{n+1 / 2,-}-V_{i}^{n} W_{i}^{n}+\tau_{n}\left(A_{i+1 / 2}^{n+1 / 4} F_{i+1 / 2}^{n+1 / 2}-A_{i-1 / 2}^{n+1 / 4} F_{i-1 / 2}^{n+1 / 2}\right) \\
= & -A_{i}^{n+1 / 4}\left(G\left(W\left(u_{i+1 / 2}^{n+1 / 2}, W_{i}^{n}, W_{i+1}^{n}\right)\right)-G\left(W\left(u_{i-1 / 2}^{n+1 / 2}, W_{i-1}^{n}, W_{i}^{n}\right)\right)\right),
\end{aligned}
$$

where $F_{2, i+1 / 2}^{n+1 / 2}$ is the Lagrangian flux:

$$
\begin{aligned}
F_{2, i+1 / 2}^{n+1 / 2} & =F_{2}\left(W\left(u_{i+1 / 2}^{n}, W_{i}^{n}, W_{i+1}^{n}\right)\right)-u_{i+1 / 2}^{n} W\left(u_{i+1 / 2}^{n}, W_{i}^{n}, W_{i+1}^{n}\right), \\
& =\left(0,0, u_{i+1 / 2}^{n} p_{i+1 / 2}^{n}, 0\right)^{T},
\end{aligned}
$$

and the terms with index (.) ${ }^{n+1 / 4}$ are defined in formula (34). As $A$ is a smooth function, we assume that $A$ is constant in a neighborhood of $x_{i+1 / 2}^{n}$. To compute $W\left(u_{i+1 / 2}^{n}, W_{i}^{n}, W_{i+1}^{n}\right)$ between two cells $C_{i}^{n}$ and $C_{i+1}^{n}$, it becomes natural to find 
an exact solution of the Riemann problem:

$$
\begin{aligned}
\partial_{t}(\rho)+\partial_{x}(\rho u) & =0, \\
\partial_{t}(\rho u)+\partial_{x}\left(\rho u^{2}+p\right) & =0, \\
\partial_{t}(\rho E)+\partial_{x}((\rho E+p) u) & =0, \\
\partial_{t}(\rho \varphi)+\partial_{x}(\rho \varphi u) & =0
\end{aligned}
$$

with the initial conditions

$$
W(x, 0)=\left\{\begin{array}{l}
W_{i}^{n}, \text { if } x<0 \\
W_{i+1}^{n}, \text { if } x>0
\end{array}\right.
$$

on the ray $\frac{x}{t}=u_{i+1 / 2}^{n}$.

With this algorithm we can compute $W_{i}^{n+1 / 2,-}$. To go back to the original grid, we use the mixed projection in the case where we have the symmetry, see Section 5.2.1, thus we obtain $W_{i}^{n+1 / 2}$ and we take $W_{i}^{n+1}=W_{i}^{n+1 / 2}$.

\subsubsection{Interface cell averaging for Glimm procedure}

As we consider Lagrangian schemes, the stability condition of the two previous schemes in Sections 5.2.1 and 5.2.2 reads

$$
\tau_{n} \leq \max _{i}\left(\frac{2 V_{i}^{n}}{A_{i-1 / 2}^{n}+A_{i+1 / 2}^{n}} \frac{1}{c_{i \pm 1 / 2}^{n}}\right) .
$$

We have also to ensure that the cell volumes do not become negative, which leads to

$$
\tau_{n} \leq \frac{1}{2} \max _{i}\left(\frac{x_{i+1 / 2}^{n}-x_{i-1 / 2}^{n}}{\left|u_{i \pm 1 / 2}\right|+c_{i \pm 1 / 2}^{n}}\right) .
$$

Then the time step $\tau_{n}$ needs to satisfy these two conditions (55) and (56).

\subsection{Numerical scheme with GFM algorithm.}

In this section, the finite volume scheme (17) has to be written in spherical coordinates. The system of Euler equations (21) written in the condensed form is:

$$
\partial_{t}(A W)+\partial_{x}(A F(W))=A^{\prime}(x) B(W),
$$

where $W$ and $F(W)$ are defined by (15) and (16), respectively. 
This system is integrated on the space-time trapezoid $Q$ as in equations (42) and (50). We end up with the following finite volume scheme

$$
\begin{array}{r}
V_{i}^{n} W_{i}^{n+1}-V_{i}^{n} W_{i}^{n}+\tau_{n}\left(A_{i+1 / 2}^{n} F_{i+1 / 2}^{n,-}-A_{i-1 / 2}^{n} F_{i-1 / 2}^{n,+}\right)= \\
\tau_{n} B\left(W_{i}^{n}\right)\left(A_{i+1 / 2}^{n}-A_{i-1 / 2}^{n}\right),
\end{array}
$$

where $V_{i}^{n}$ is given by (30). The fluxes $F_{i+1 / 2}^{n,-}$ and $F_{i-1 / 2}^{n,+}$ are determined by the procedure of Section 3. For more details on the derivation of the discretization, we refer to [MBKKH09].

\subsection{Numerical results}

We compare the three above algorithms presented in Sections 5.2 and 5.3 by means of the oscillation of a spherical bubble. We study the "weakly" compressed bubble and the more difficult test case of the "strongly" compressed bubble. In the two case we start the computation at time $t=0 \mathrm{~s}$ when the radius of the bubble is maximal, i.e. $R_{b}=R_{\max }$. We have to choose the size of the domain such that the right wave does not have time to catch the right side of the domain. In the weakly compressed bubble this condition is not so restrictive because the speed of sound in the liquid phase is not so large $\left(c_{l i q}=50 \mathrm{~m} . \mathrm{s}^{-1}\right)$. For the strongly compressed bubble $\left(c_{l i q}=1500 \mathrm{~m}_{\mathrm{s}} \mathrm{s}^{-1}\right)$, this condition imposes to choose a large domain to avoid interactions with reflections coming from the right boundary. For these two test cases we will study by front tracking, the radius of the spherical bubble of gas. We compare our results to the model of Keller-Miksis, see [KM80, MBKKH09], This is an ODE model that gives an approximate solution of the bubble radius for a given equilibrium radius $R_{e q}$. In our case, $R_{e q}=0.692 \times 10^{-4} \mathrm{~m}$ that fits the model to the experimental results of the strongly compressed bubble.

\subsubsection{The weakly compressed bubble}

In this case the maximal radius of the bubble is $R_{\max }=0.7469 \times 10^{-4} \mathrm{~m}$. Initial states and material parameters computed in Section 5.1.2 are recalled in Table 7 and Table 8 . The computational domain is $\left[0 ; 20 R_{b}\right]$ and the final time is $t_{\text {final }}=5.5 e-5 \mathrm{~s}$. 
[Table 7 about here.]

[Table 8 about here.]

The result on the evolution of the radius of the bubble is presented in Figure 14 .

[Figure 14 about here.]

Note that the two algorithms "Glimm with $p D_{x} A$ " and "Glimm with $A D_{x} p$ ", presented in Section 5.2.1 and Section 5.2.2, respectively, are two different methods to study the source term of the system (21)-(22). These two algorithms have been performed on a uniform mesh of 16000 points. They give approximatively the same result. After the first rebound, the results become different to the GFM model which is more similar to the Keller-Miksis model. Moreover, contrary to the Keller-Miksis model, our algorithms provide us with all quantities at each time step. Thus, we choose to plot the pressure and the velocity when the bubble shrinks and expands again for the same radius $R_{1}=6.8 \times 10^{-5} \mathrm{~m}$, i.e., at time $t=6.47 \times 10^{-6} \mathrm{~s}$ and $t=1.37 \times 10^{-5} \mathrm{~s}$, respectively. The results are presented in Figures 15 and 16. The three algorithms give the same result for the velocity, see Figure 15, when the bubble collapses the velocity inside the bubble is negative and when the bubble expands this velocity is positive. In the pressure graph, there is a small difference between both algorithms with Glimm procedure and the GFM. For the GFM the pressure at the center of the bubble is a little bit larger than for both algorithms with Glimm procedure, see Figure 16. However, the shape of the graph is the same, the pressure inside the bubble is higher than the pressure outside the bubble.

We can see in Figures 15 and 16 that we have no oscillations in the pressure and velocity at the interface.

[Figure 15 about here.]

[Figure 16 about here.] 


\subsubsection{The strongly compressed bubble}

This test case corresponds to the numerical test where the maximal radius of the bubble is $R_{\max }=0.7469 \times 10^{-3} \mathrm{~m}$. Initial states and material parameters computed in Section 5.1.2 are recalled in Table 9 and Table 10. The computational domain is $[0 ; 0.1]$, because we need to choose a larger domain if we do not want that the reflections on the right boundary interact with the interface. The final time is $t_{\text {final }}=1.6 \mathrm{~ms}$.

[Table 9 about here.]

[Table 10 about here.]

As the gas and the water have extremely different physical properties and as the difference in pressure inside and outside the bubble is huge, this case is very difficult to study. In fact, most of classical algorithms (Godunov scheme or averaging projection for example) do not work on this test. Moreover, as the bubble becomes very small, we have to choose the space discretization such that it stays smaller than the minimal radius of the bubble, i.e, we need to use a fine mesh.

As the computational domain is huge and as we need a fine mesh at the interface bubble, the computational time for both algorithms using Glimm projection explode if we use a uniform mesh. We choose to cut the grid refinement on $x_{\text {rup }}=2 \times R_{\max }$ and we take a uniform mesh with 8000 points on the right side of $x_{\text {rup }}$ and 8000 point on the left side of $x_{\text {rup }}$. The results are presented in Figure 17. The "GFM" computation was performed on an adaptively refined mesh, where the number of coarse grid cells is $N_{0}=100$ and the number of refinement levels is $L=12$.

[Figure 17 about here.]

We see in Figure 17 that the amplitude of the first rebound for the three algorithms is comparable but smaller than the one of the Keller-Miksis model. More precisely, the "Glimm with $A D_{x} p$ " approach has the better first rebound with 
an amplitude corresponding to $72 \%$ of the one of the Keller-Miksis model. Note that the parameters of the Keller-Miksis model are chosen such that it fits to the experimental results of a laser-induced single bubble collapse, see [MBKKH09]. For the GFM and the "Glimm with $p D_{x} A$ ", they are at $65 \%$ and $62,7 \%$, respectively. Moreover, the GFM presents a little shift in the first rebound referred to the others.

However, for the other rebounds, the results are more different. With the "Glimm with $p D_{x} A$ " algorithm and the GFM, the damping in the next rebounds is more important than with "Glimm with $A D_{x} p$ " algorithm which is very close to the Keller-Miksis model although with a smaller amplitude. In the "Glimm with $p D_{x} A^{\prime \prime}$ approach and the GFM, the same set of equations are solved that could explain the similarity in the rebounds.

For better results we have to pass to the second order as it is done in [MBKKH09] for the GFM. Both algorithms using Glimm procedure do not smear the interface (resolved with $\varphi$ ) between the gas and the liquid phase, it could be possible to pass to second order in space with a method of MUSCL type. We cannot pass to second order in time if we do not want to smear the interface. With a second order space approximation and a first order time approximation, the algorithm would be too unstable on refined meshes.

Remark 2. In this case, we do not plot all quantities at different times because we have a pressure jump at the interface with both algorithms using Glimm projection. But there is no problem with the velocity. We do not really found an explanation to this phenomena. We plot the velocity, see Figure 18, and the pressure, see Figure 19, at two different times $t_{1}=1.3 \times 10^{-7} \mathrm{~s}$ and $t_{2}=1.3 \times 10^{-5} \mathrm{~s}$, before the first movement of the interface with Glimm. We note that for a very short time $t=t_{1}$, it seems to be a regularization of the pressure between the two initial states. However, at time $t_{2}$ a pressure jump appears.

This problem does not come from the conservation error of the Glimm projection. To check this we tried a pure Lagrangian scheme, i.e., the boundary of the cell moves and we do not need to perform a projection. We tested this with the same final time $t_{2}$ and obtained exactly the same result as with the Lagrange-projection scheme, i.e., the same pressure jump.

[Figure 18 about here.]

[Figure 19 about here.] 


\section{Conclusion}

In this paper, we have proposed a new Lagrangian-projection scheme for computing two-fluid flows. The pressure oscillations at the interface are avoided thanks to a Lagrange and a projection approach. In the Lagrange step, the contact waves are perfectly resolved and the interface is not smeared. In the projection step, we employ a random sampling strategy. The resulting scheme preserves the constant velocity-pressure states and the interface is solved by one grid point.

The whole approach performs well for weak shocks. But in the presence of strong shocks, it appears to be oscillating. Therefore, we had to adapt the projection step and only apply it at the two-fluid interface, which is located thanks to the jumps of the colour function. We then presented numerical results that demonstrate the good convergence behaviour of the scheme, despite that it is not conservative. We surprisingly observed this convergence property for other non-conservative schemes for two-fluid flows.

Finally, we apply our Lagrange-projection scheme to a more challenging problem, which consists in the simulation of the oscillations of a single gas bubble in a compressible liquid. In the weakly compressed case, all methods give good results, i.e., in the amplitude of the rebounds and in the time instant of the collapses. In the strongly compressed case, the first collapse instant is shifted in time for the GFM compared to the others and the Keller-Miksis model and the damping in the first rebound is more important than for the Keller-Miksis model. Nevertheless, the damping for the mixed projection with the $A D_{x} p$ algorithm in the next rebounds is very close to the Keller-Miksis model. The use of a first order scheme in space being the explanation of the damping and the shift in time of the first collapse for the GFM, the use of a second order scheme should improve the results for the Lagrangian algorithms.

Our perspectives are in several directions:

- first we would like to improve the precision of the random projection scheme. The first obvious way to do it is to couple it with a second 
order MUSCL extension. This extension has to be deactivated at the interface, in order to avoid oscillations. For the spherical bubble computations, another way to improve the precision is to modify the scheme such that it becomes well-balanced. This can be done by adapting the method described in [HHM10].

- a challenging extension would consist in extending the random projection scheme to two- or three-dimensional computations, for instance by a simple directional splitting algorithm. This will be the objective of a forthcoming work.

\section{References}

[Abg88] R. Abgrall. Generalisation of the Roe scheme for the computation of mixture of perfect gases. Recherche Aérospatiale, 6 (1988), 31-43.

[AK01] R. Abgrall, S. Karni. Compressible multifluid flows. J. Comp. Phys., 169 (2001), 594-623.

[AK10] R. Abgrall, S. Karni. A comment on the computation of nonconservative products. J. Comp. Phys., 229 (2010), 2759-2763.

[BAB86] M. Ben-Artzi, A. Birman. Application of the "generalized Rimann problem" method to $1 \mathrm{D}$ compressible flows with material interfaces. J. Comp. Phys., 65 (1986), 170-178.

[BA02] T. Barberon. Modélisation mathématique et numérique de la cavitation dans les écoulements multiphasiques compressibles. Phd thesis, Université de Toulon et du Var, 2002.

[BHR03] T. Barberon, P. Helluy, and S. Rouy. Practical computation of axisymmetrical multifluid flows. International Journal of Finite Volumes, 1 (2003), no. 1, 1-34. 
[CC08] C. Chalons, F. Coquel. Capturing infinitely sharp discrete shock profiles with the Godunov scheme. Hyperbolic problems: theory, numerics, applications, 363-370, Springer, Berlin, 2008.

[CG07] C. Chalons, P. Goatin. Transport-equilibrium schemes for computing contact discontinuities in traffic flow modeling. Commun. Math. Sci., 5 (2007), no. $3,533-551$.

[FAMO99] R.P. Fedkiw, T. Aslam, B. Merriman, S. Osher. A non-oscillatory Eulerian approach to interfaces. J. Comp. Phys., 152 (1999), no. 2, $457-492$.

[FGG01] C. Farhat, P. Geuzaine, C. Grandmont. The discrete geometric conservation law and the nonlinear stability of the ALE schemes for the solution of flow problems on moving grids. J. Comp. Phys., 174 (2001), no. $2,669-694$.

[FRS08] C. Farhat, A. Rallu, S. Shankaran. A higher-order generalized ghost fluid method for the poor for the three-dimensional two-phase flow computation of underwater implosions. J. Comp. Phys., 227 (2008), $7674-7700$.

[Gl65] J. Glimm. Solutions in the large for nonlinear hyperbolic systems of equations. Comm. Pure Appl. Math., 18 (1965), 697-715.

[GOD96] E. Godleski, P.-A. Raviart. Numerical approximation of hyperbolic systems of conservation laws. Applied Mathematical Sciences, Springer-Verlag, vol. 118, New York, 1996.

[HHM10] P. Helluy, J.-M. Hérard, H. Mathis. A well-balanced approximate Riemann solver for variable cross-section compressible flows. AIAA Proc. 22-25 Jun, 2009.

[HMM08] P. Helluy, H. Mathis, S. Müller. An ALE averaging approach for the computing of bubble oscillations. Finite volumes for complex applications V, 487-494, ISTE, London, 2008. 
[HL94] T. Y. Hou, P.G. LeFloch. Why nonconservative schemes converge to wrong solutions: error analysis. Math. Comp. 62 (1994), no. 206, 497-530.

[Kar94] S. Karni. Multicomponent flow calculations by a consistent primitive algorithm. J. Comp. Phys., 112 (1994), no. 1, 31-43.

[KM80] J.B. Keller, M. Miksis. Bubble oscillations of large amplitude. J. Acoust. Soc. Am., 68 (1980), no. 2, 628-633.

[KL10] S. Kokh, F. Lagoutière. An anti-diffusive numerical scheme for the simulation of interfaces between compressible fluids by means of a five-equation model. J. Comp. Phys., 229 (2010), no. 8, 2773-2809.

[PL86] A. Prosperetti, A. Lezzi. Bubble dynamics in a compressible liquid. I. First-order theory. J. Fluid Mech., 168, pp: 457-478, 1986.

[Ma10] H. Mathis. Étude théorique et numérique des écoulements avec transition de phase. PhD thesis, Université de Strasbourg, 2010. http: //tel.archives-ouvertes.fr/IRMA/tel-00516683/fr/

[MÜ03] S. Müller. Adaptive multiscale schemes for conservation laws. Lecture Notes in Computational Science Engineering, Springer, vol 27, 2003.

[MBKKH09] S. Müller, M. Bachmann, D. Kröninger, T. Kurz, P. Helluy. Comparison and validation of compressible flow simulations of laserinduced cavitation bubbles. Computers \& Fluids. 38 (2009), no. 9, $1850-1862$.

[SA99a] R. Saurel, R. Abgrall. A simple method for compressible multifluid flows. SIAM J. Sci. Comput., 21 (1999), no. 3, 1115-1145.

[SA99b] R. Saurel, R. Abgrall. A multiphase Godunov method for compressible multifluid and multiphase flows. J. Comp. Phys., 150 (1999), no. $2,425-467$. 
[Tor99] E.F. Toro. Riemann solvers and numerical methods for fluid dynamics. A practical introduction. Second edition. Springer-Verlag, Berlin, 1999.

[WK05] J. Wackers, B. Koren. A fully conservative model for compressible two-fluid flow. 8th ICFD Conference on Numerical Methods for Fluid Dynamics. Part 2. Internat. J. Numer. Methods Fluids, 47 (2005), no. 10-11, 1337-1343.

[WLK06] C. W. Wang, T. G. Liu, B. C. Khoo. A real ghost fluid method for the simulation of multimedium compressible flow. SIAM J. Sci. Comput., 28 (2006), no. 1, 278-302. 


\section{List of Figures}

1 Sketch of the computation of the real and ghost fluid states from the interfacial states $u_{I}, p_{I}$ and $\rho_{I L}, \rho_{I R}$ determined by solving a two-phase Riemann problem for the states $u_{L}$ and $u_{R} \ldots \ldots 39$

2 Academic validation: Convergence study. Averaging projection, Glimm projection, Saurel-Abgrall approach and ghost fluid method. 40

3 Shock-interface interaction: Wave structure at different times. . 41

4 Academic shock-interface interaction: Glimm approach, density plot. . . . . . . . . . . . . . . . . . . 4 42

5 Academic shock-interface interaction: Density. Comparison of the mixed and averaging projection schemes. . . . . . . . 43

6 Academic shock-interface interaction: Density profiles for the exact solution and the approximation using the Saurel-Abgrall approach (S-A) and the ghost fluid method (GFM). . . . . . . . 44

7 Academic shock-interface interaction. Convergence study. . . . . 45

8 Water-Air shock-interface interaction: Results at $t_{A}=1.5 \mathrm{~ms}$ with a finest resolution of $470 \mu \mathrm{m}(L=7) \ldots \ldots \ldots \ldots$

9 Water-Air shock-interface interaction: Results for $L=12$ refinement levels at $t_{A}=1.5 \mathrm{~ms} . \ldots \ldots \ldots . \ldots . \ldots 47$

10 Water-Air shock-interface interaction: Results for $L=7$ refine-

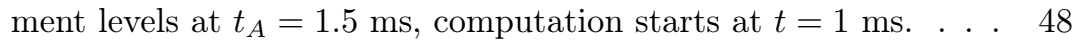

11 Water-Air shock-interface interaction: Convergence of the $L^{1}$ error in the interface position. . . . . . . . . . . . . . . . 49

12 Water-Air shock-interface interaction: Convergence of the interface position error. . . . . . . . . . . . . 50

13 Illustration of the test of the bubble. . . . . . . . . . . 51

14 Test of the bubble: Oscillation of the radius of the weakly compressed bubble. . . . . . . . . . . . . . . . . 52

15 Velocity for the weakly compressed bubble when the radius is equal to $R_{1}$ at time $t_{1}=6.47 \times 10^{-6} \mathrm{~s}$ (left) and $t_{2}=1.37 \times 10^{-5} \mathrm{~s}$

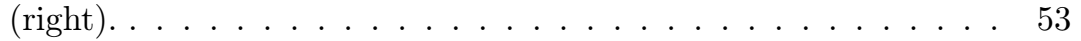

16 Pressure for the weakly compressed bubble when the radius is equal to $R_{1}$ at time $t_{1}=6.47 \times 10^{-6} \mathrm{~s}$ (left) and $t_{2}=1.37 \times 10^{-5} \mathrm{~s}$

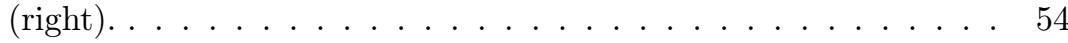

17 Test of the bubble: Oscillation of the radius of the strongly compressed bubble. . . . . . . . . . . . . . 55

18 Velocity for the strongly compressed bubble at time $t_{1}=1.3 \times$ $10^{-7} s$ (left) and $t_{2}=1.3 \times 10^{-5} s$ (right). . . . . . . 56

19 Presure for the strongly compressed bubble at time $t=1.3 \times$

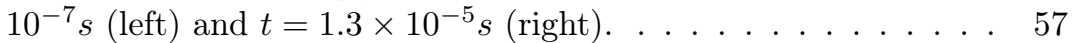




\begin{tabular}{l}
\multicolumn{2}{c}{ Fluid A } \\
\hline \\
\hline \\
\hline
\end{tabular}

Figure 1: Sketch of the computation of the real and ghost fluid states from the interfacial states $u_{I}, p_{I}$ and $\rho_{I L}, \rho_{I R}$ determined by solving a two-phase Riemann problem for the states $u_{L}$ and $u_{R}$. 


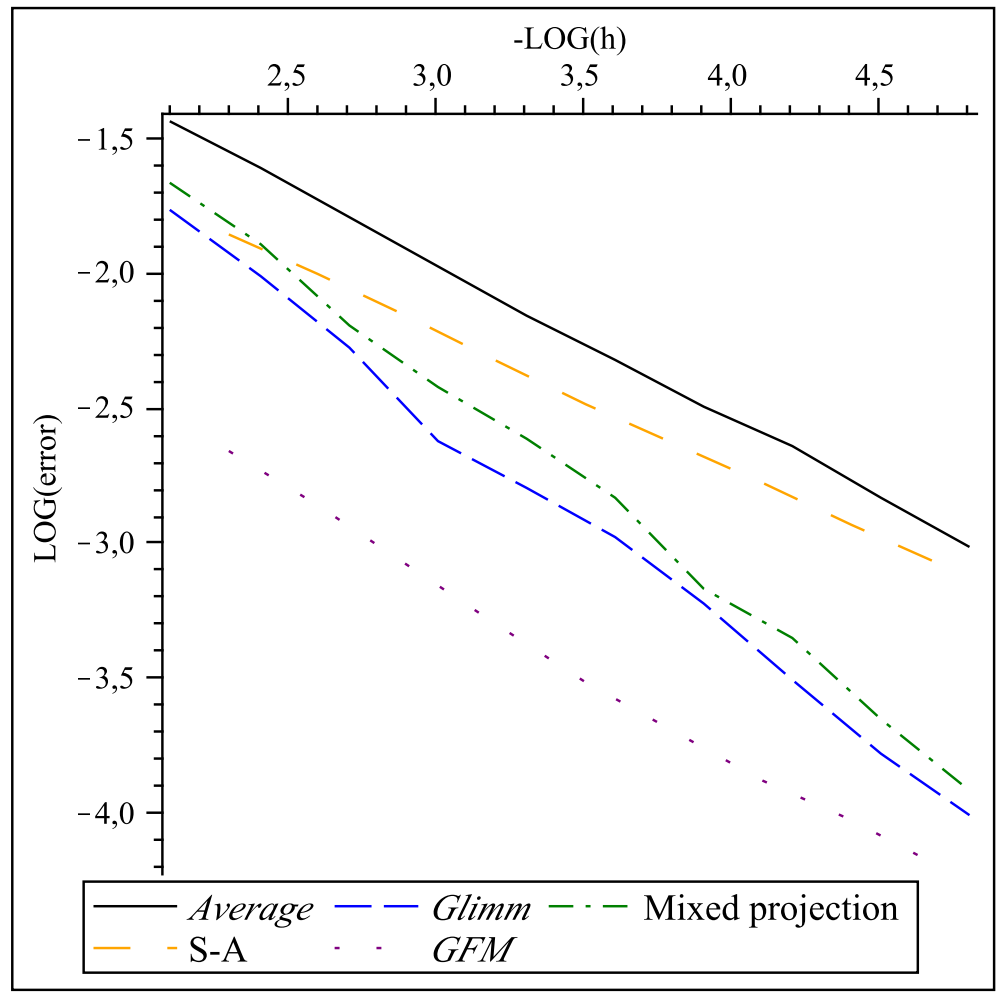

Figure 2: Academic validation: Convergence study. Averaging projection, Glimm projection, Saurel-Abgrall approach and ghost fluid method. 
Configuration at initial time $t_{0}$

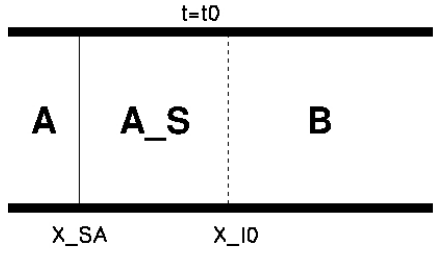

Configuration at interaction instant $t_{I}$

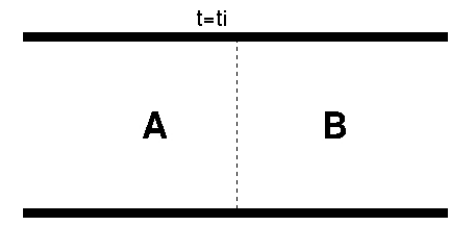

x_s

Configuration after interaction at $t_{A}$

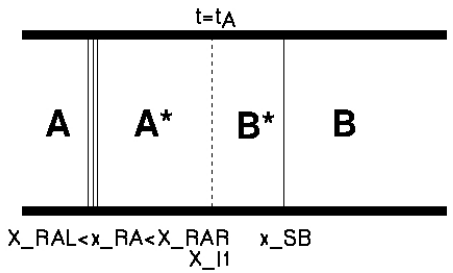

Figure 3: Shock-interface interaction: Wave structure at different times. 


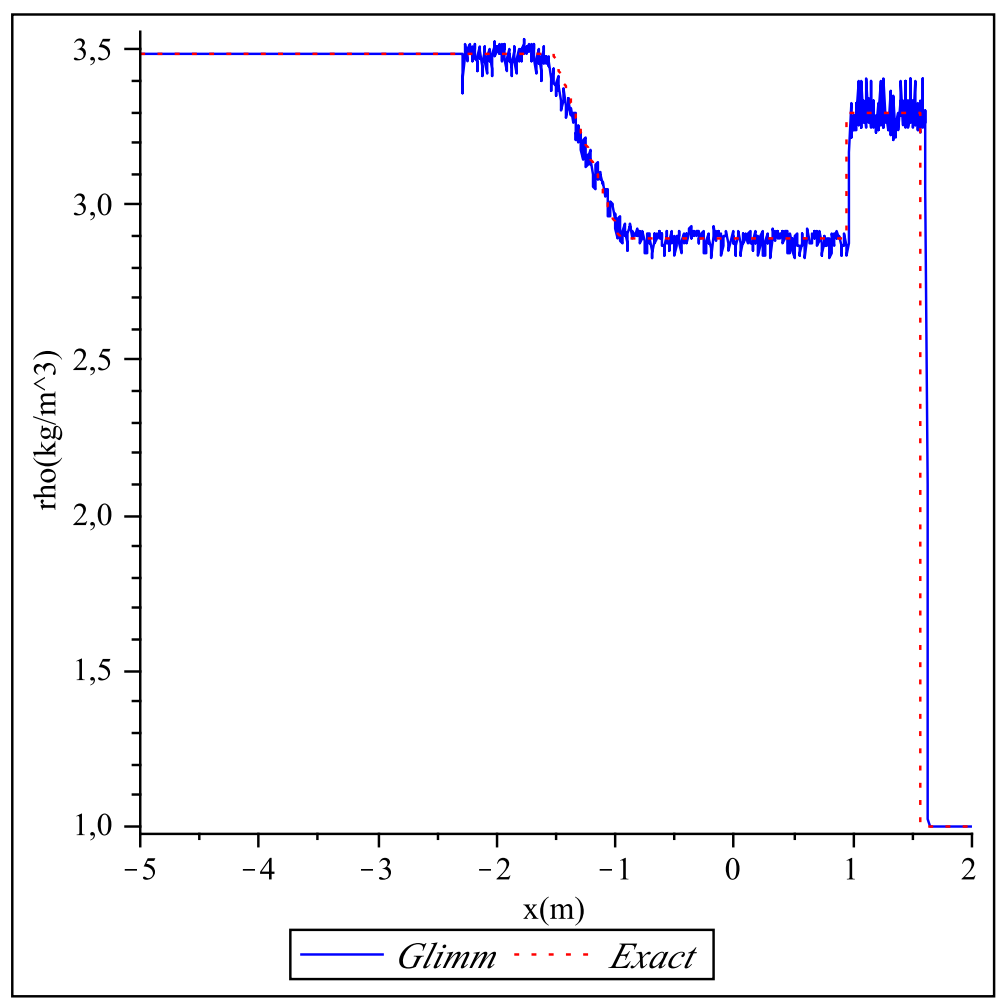

Figure 4: Academic shock-interface interaction: Glimm approach, density plot. 


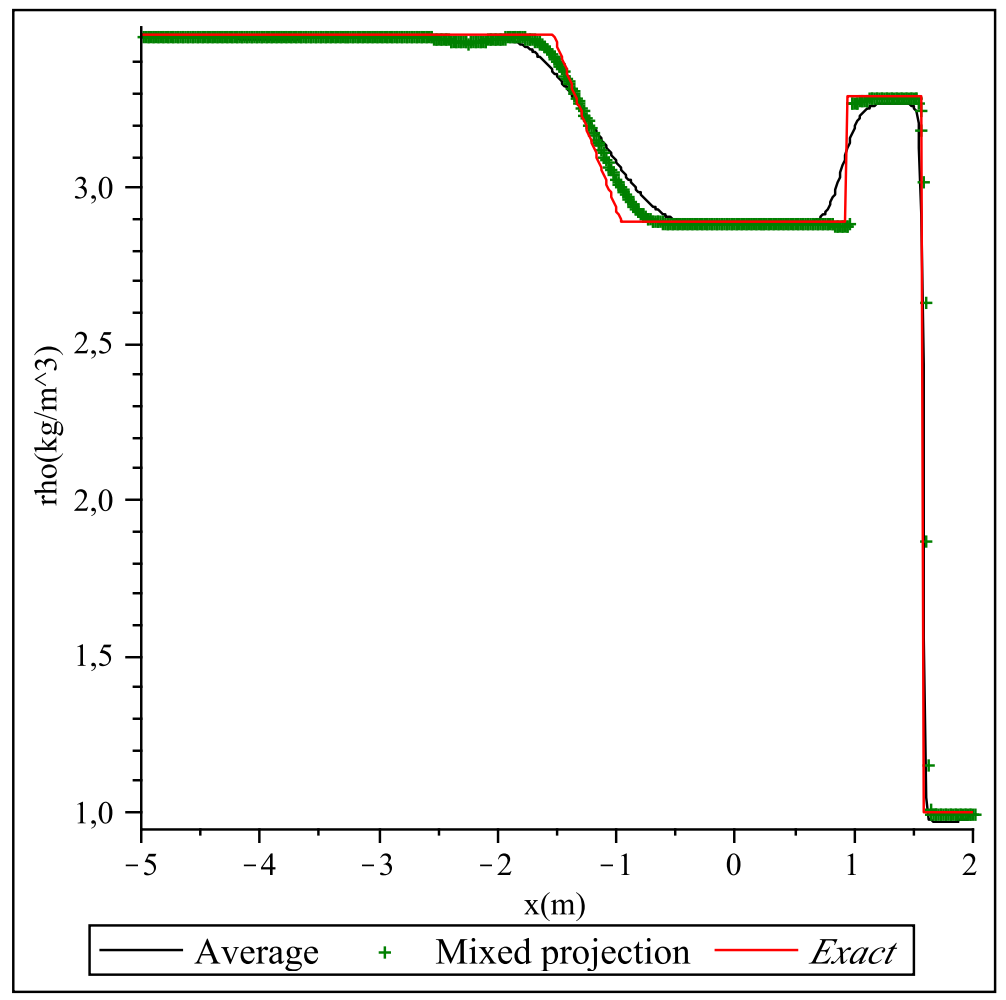

Figure 5: Academic shock-interface interaction: Density. Comparison of the mixed and averaging projection schemes. 


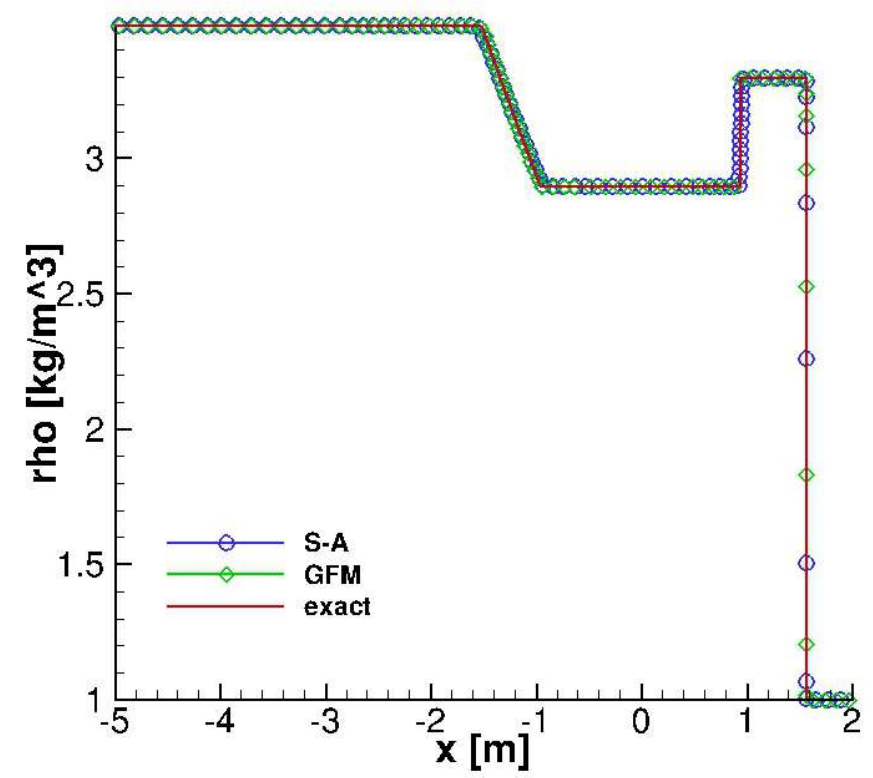

Figure 6: Academic shock-interface interaction: Density profiles for the exact solution and the approximation using the Saurel-Abgrall approach (S-A) and the ghost fluid method (GFM). 


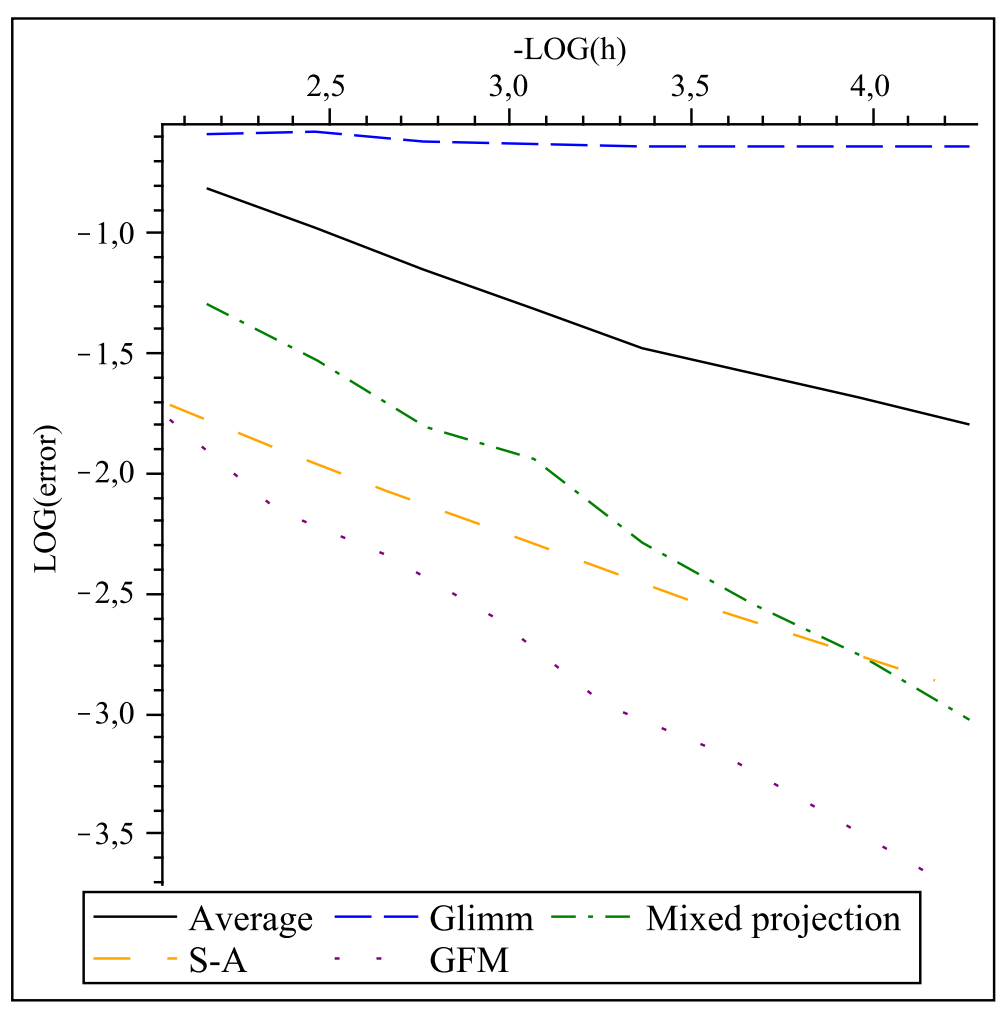

Figure 7: Academic shock-interface interaction. Convergence study. 


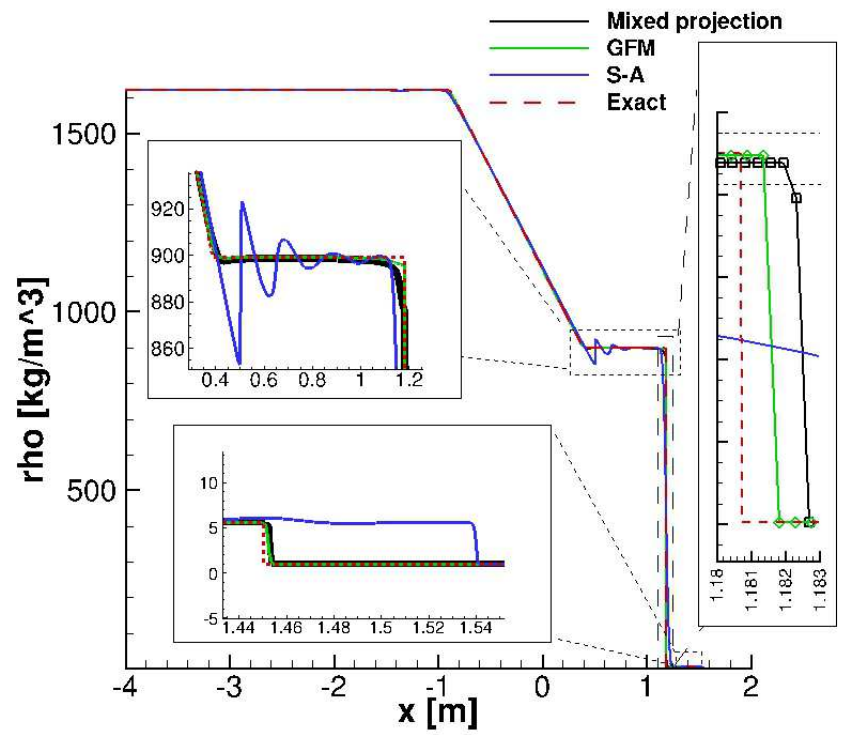

Figure 8: Water-Air shock-interface interaction: Results at $t_{A}=1.5 \mathrm{~ms}$ with a finest resolution of $470 \mu \mathrm{m}(L=7)$. 


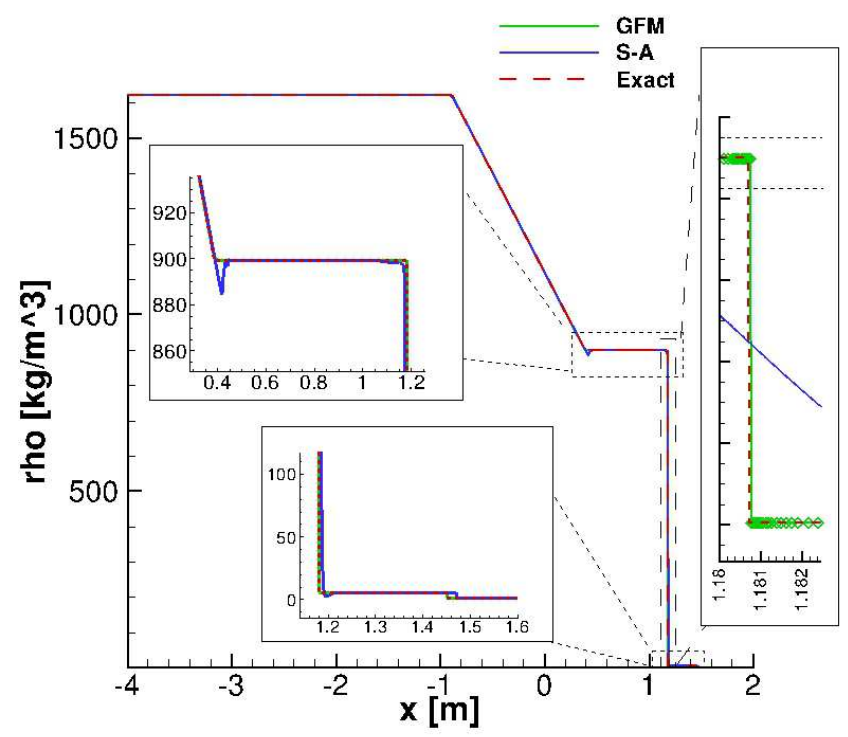

Figure 9: Water-Air shock-interface interaction: Results for $L=12$ refinement levels at $t_{A}=1.5 \mathrm{~ms}$. 


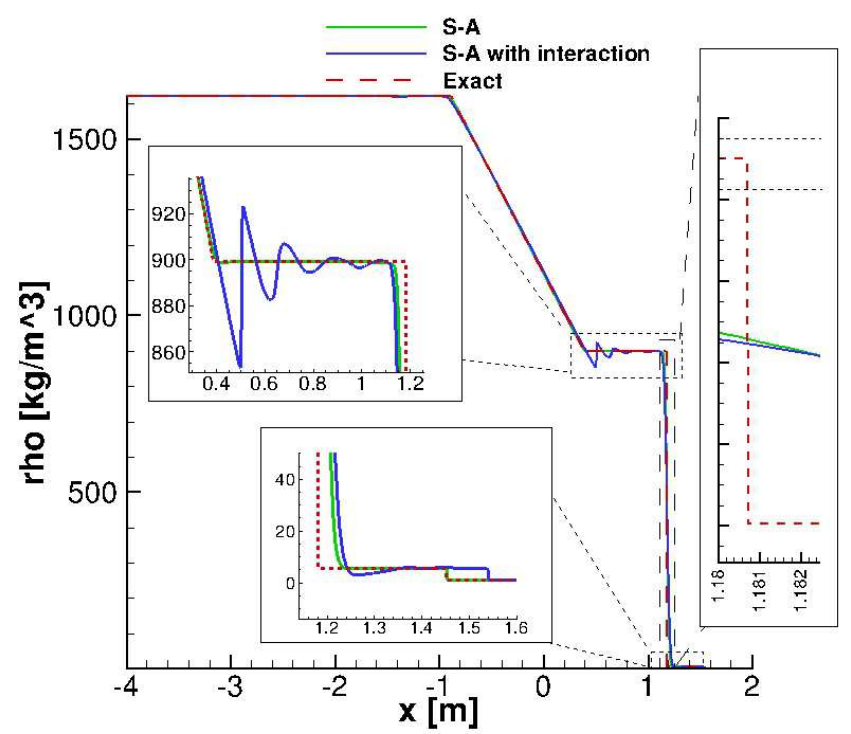

Figure 10: Water-Air shock-interface interaction: Results for $L=7$ refinement levels at $t_{A}=1.5 \mathrm{~ms}$, computation starts at $t=1 \mathrm{~ms}$. 


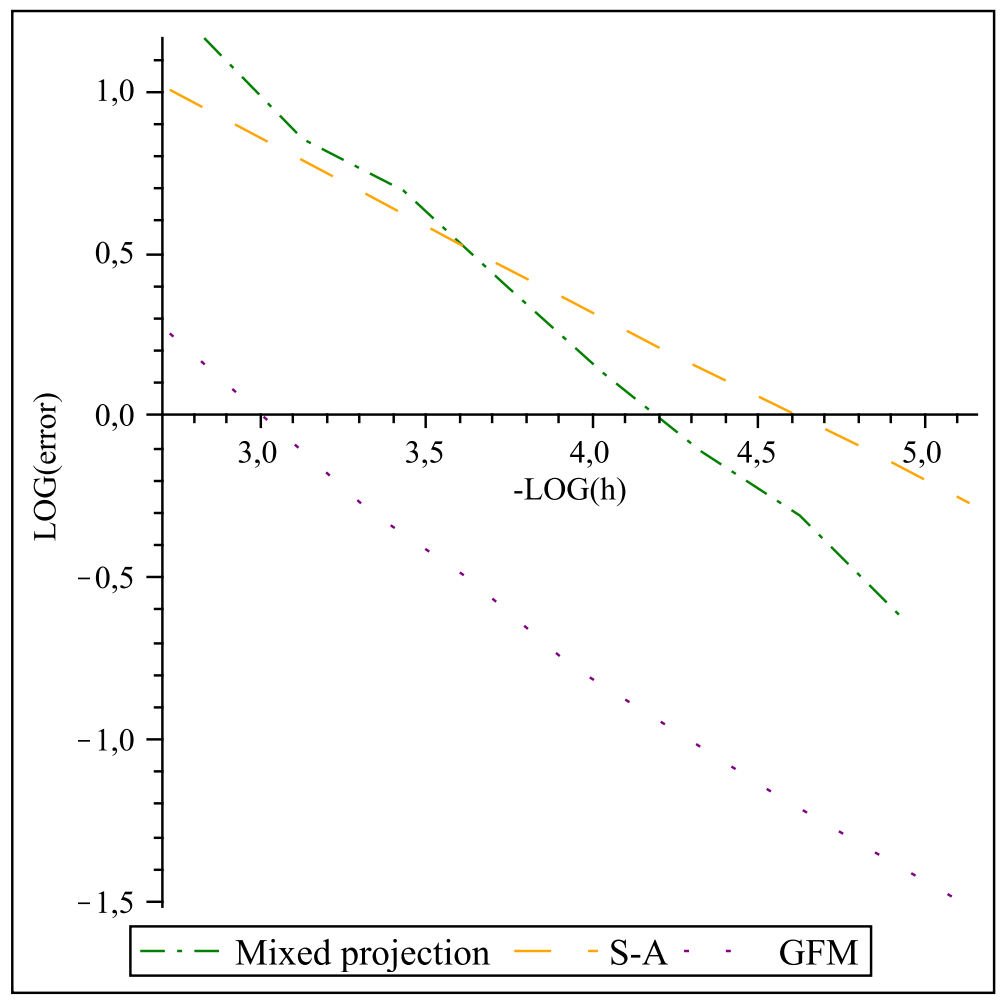

Figure 11: Water-Air shock-interface interaction: Convergence of the $L^{1}$-error in the interface position. 


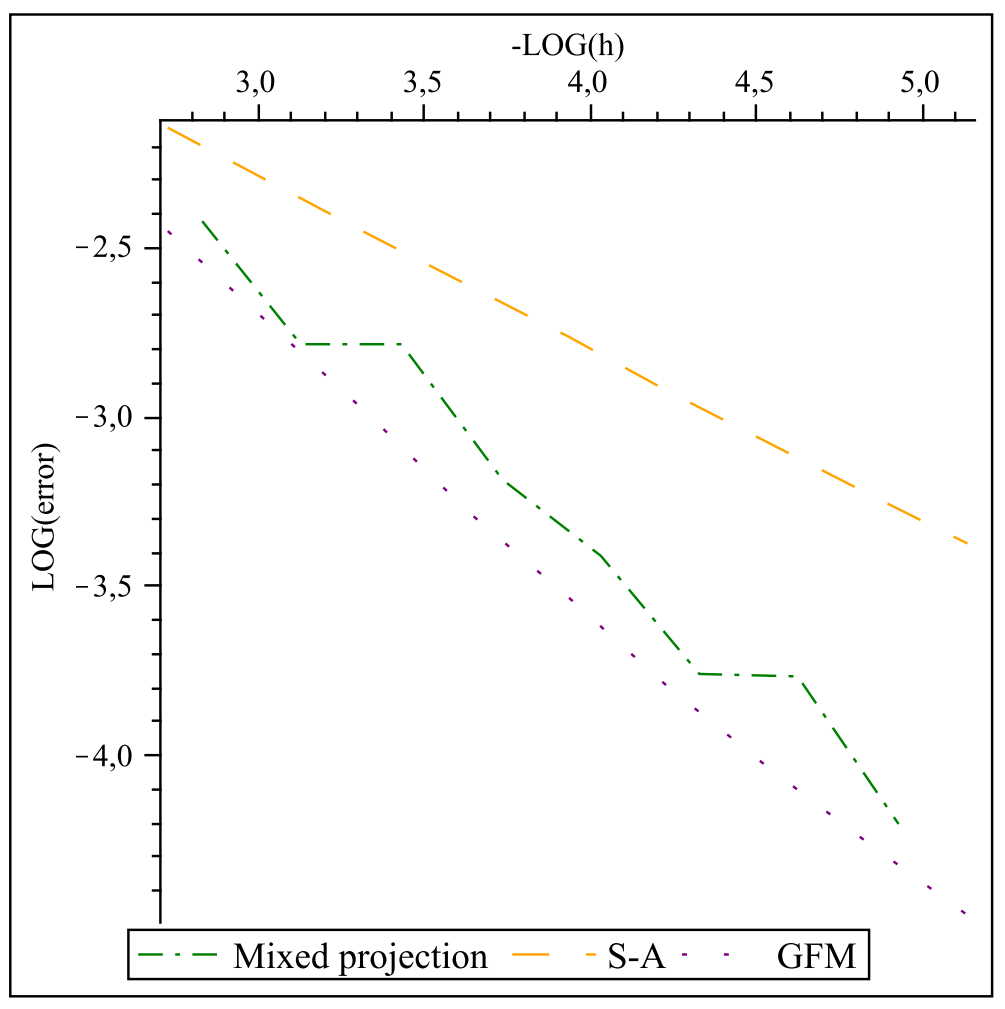

Figure 12: Water-Air shock-interface interaction: Convergence of the interface position error. 


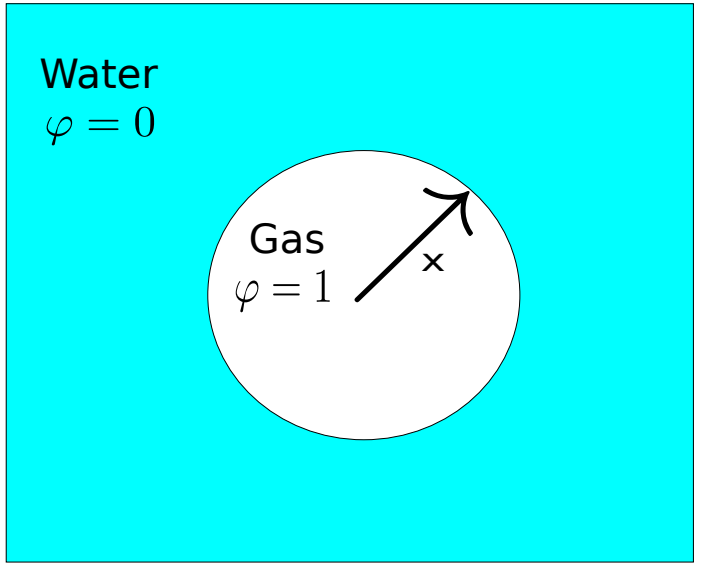

Figure 13: Illustration of the test of the bubble. 


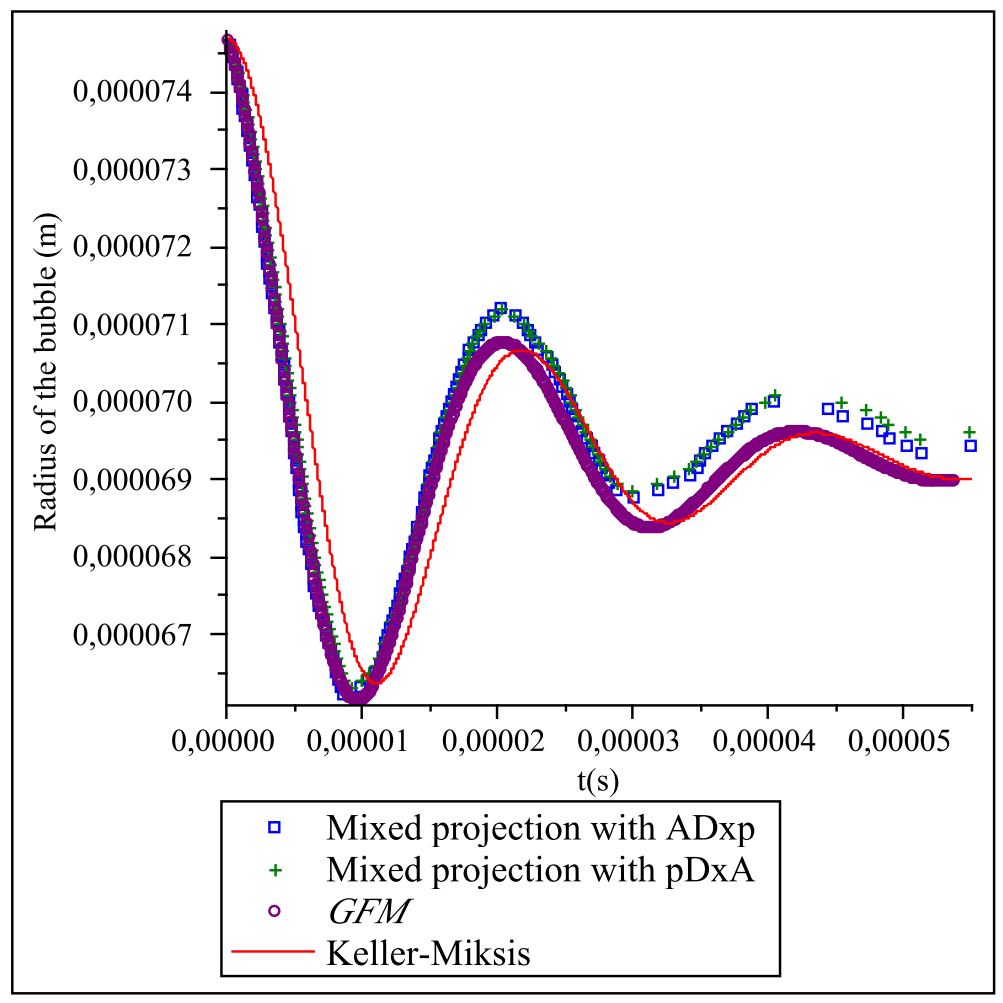

Figure 14: Test of the bubble: Oscillation of the radius of the weakly compressed bubble. 

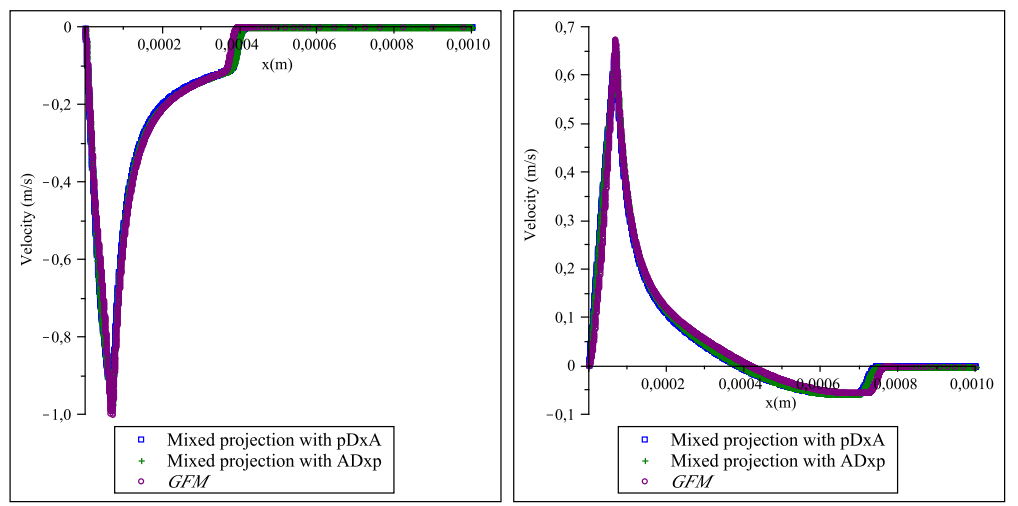

Figure 15: Velocity for the weakly compressed bubble when the radius is equal to $R_{1}$ at time $t_{1}=6.47 \times 10^{-6} \mathrm{~s}$ (left) and $t_{2}=1.37 \times 10^{-5} \mathrm{~s}$ (right) . 

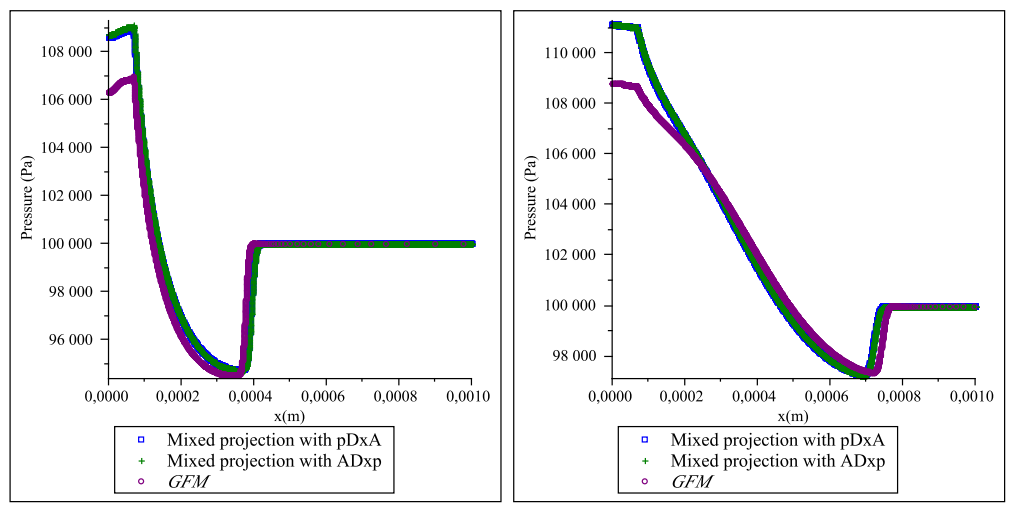

Figure 16: Pressure for the weakly compressed bubble when the radius is equal to $R_{1}$ at time $t_{1}=6.47 \times 10^{-6} \mathrm{~s}$ (left) and $t_{2}=1.37 \times 10^{-5} \mathrm{~s}$ (right). 


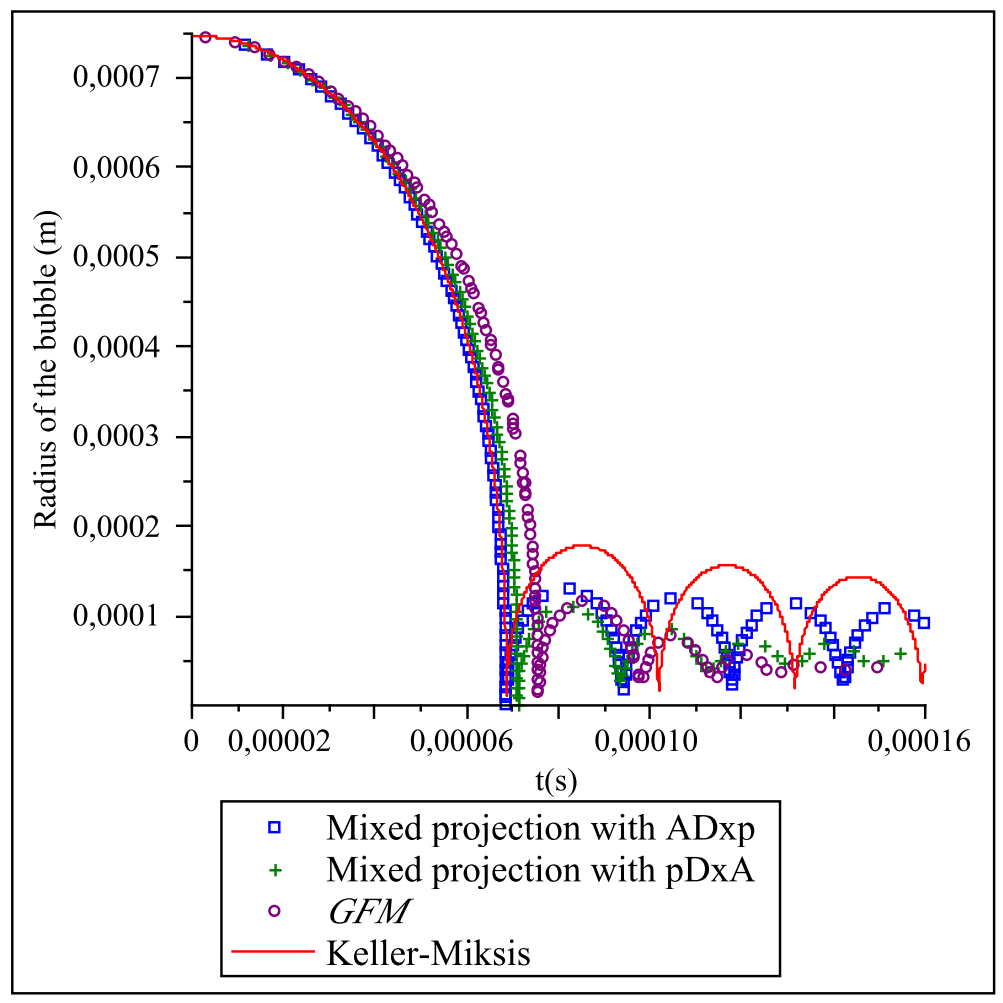

Figure 17: Test of the bubble: Oscillation of the radius of the strongly compressed bubble. 

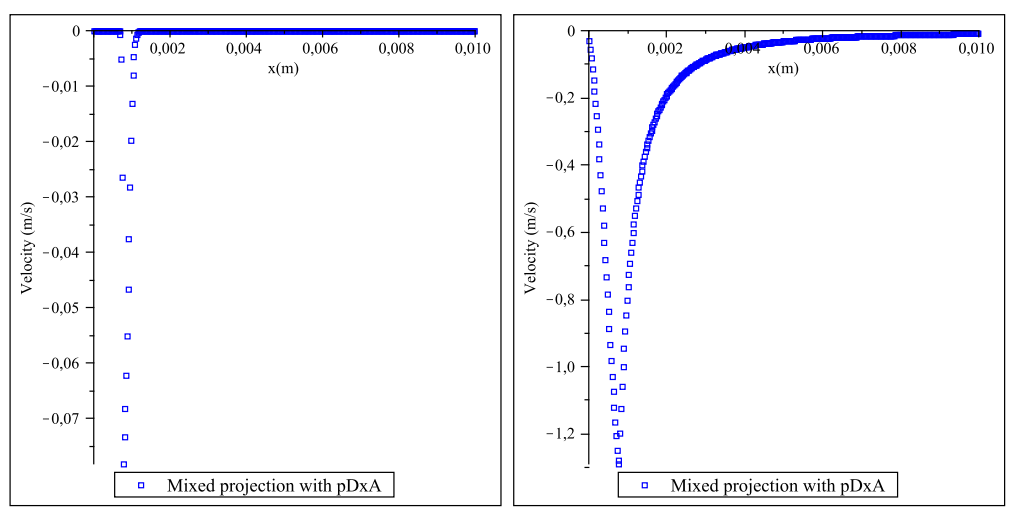

Figure 18: Velocity for the strongly compressed bubble at time $t_{1}=1.3 \times 10^{-7} \mathrm{~s}$ (left) and $t_{2}=1.3 \times 10^{-5} s$ (right). 

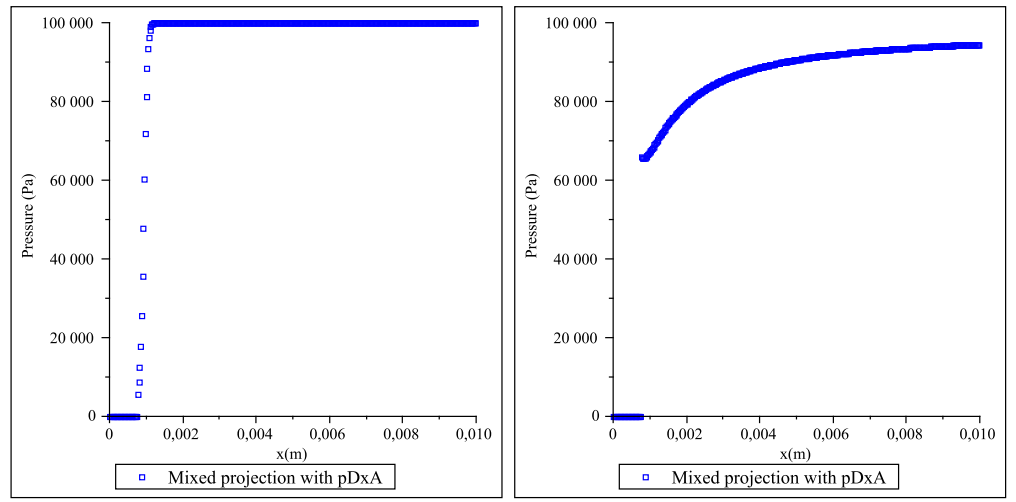

Figure 19: Presure for the strongly compressed bubble at time $t=1.3 \times 10^{-7} \mathrm{~s}$ (left) and $t=1.3 \times 10^{-5} s$ (right). 


\section{List of Tables}

1 Academic validation: Material parameters. . . . . . . . . . 59

2 Academic validation: initial left and right states. . . . . . . . 60

3 Academic shock-interface interaction: States at different times in

Fig. 3. . . . . . . . . . . . . . . . . . . 61

4 Academic shock-interface interaction: Material parameters. . . . 62

$5 \quad$ : States at different times in Fig. 3. . . . . . . . . . . . 63

6 Water-Air shock-interface interaction: Material parameters of the stiffened gas law for water and air. . . . . . . . . . . . . . 64

$7 \quad$ Weakly compressed gas bubble: Initial left and right states. . . . 65

8 Weakly compressed gas bubble: Material parameters. . . . . . . 66

$9 \quad$ Strongly compressed gas bubble: Initial left and right states. . . 67

10 Strongly compressed gas bubble: Material parameters. . . . . . . 68 


\begin{tabular}{|l|l|l|}
\hline & $\gamma[-]$ & $\pi[\mathrm{Pa}]$ \\
\hline Fluid 2 & 2.0 & 1 \\
Fluid 1 & 1.4 & 0 \\
\hline
\end{tabular}

Table 1: Academic validation: Material parameters. 


\begin{tabular}{|l|l|l|}
\hline & $W_{L}$ & $W_{R}$ \\
\hline$\rho\left[\mathrm{kg} / \mathrm{m}^{3}\right]$ & 2 & 1 \\
$u[\mathrm{~m} / \mathrm{s}]$ & 0.5 & 0.5 \\
$p[\mathrm{~Pa}]$ & 2 & 1 \\
$\varphi$ & 1 & 0 \\
\hline
\end{tabular}

Table 2: Academic validation: initial left and right states. 


\begin{tabular}{|l|l|l|l|l|l|}
\hline & $W_{A}$ & $W_{A S}$ & $W_{A *}$ & $W_{B *}$ & $W_{B}$ \\
\hline$\rho\left[\mathrm{kg} / \mathrm{m}^{3}\right]$ & 3.488 & 2 & 2.89415 & 3.2953 & 1 \\
$u[\mathrm{~m} / \mathrm{s}]$ & 1.13 & -1 & 1.87672 & & -1 \\
$p[\mathrm{~Pa}]$ & 23.33 & 2 & 13.88 & 13.88 & 2 \\
$\varphi$ & 1 & 1 & - & - & 0 \\
\hline
\end{tabular}

Table 3: Academic shock-interface interaction: States at different times in Fig. 3. 


\begin{tabular}{|l|l|l|}
\hline & $\gamma[-]$ & $\pi[\mathrm{Pa}]$ \\
\hline Fluid A & 2 & 7 \\
Fluid B & 1.4 & 0 \\
\hline
\end{tabular}

Table 4: Academic shock-interface interaction: Material parameters. 


\begin{tabular}{|l|l|l|l|l|l|}
\hline & $W_{A}$ & $W_{A S}$ & $W_{A *}$ & $W_{B *}$ & $W_{B}$ \\
\hline$\rho\left[\mathrm{kg} / \mathrm{m}^{3}\right]$ & 1620.6 & 1000 & 900 & 5.57 & 1 \\
$u[\mathrm{~m} / \mathrm{s}]$ & 1087.1 & -100 & 2361.4 & 2361.4 & -100 \\
$p[\mathrm{~Pa}]$ & $3.6801 \mathrm{E}+09$ & $1 \mathrm{E}+05$ & $7.48506 \mathrm{E}+06$ & $7.48506 \mathrm{E}+06$ & $1 \mathrm{E}+05$ \\
$\varphi$ & 1 & 1 & - & - & 0 \\
\hline
\end{tabular}

Table 5: : States at different times in Fig. 3. 


\begin{tabular}{|l|l|l|}
\hline & $\gamma[-]$ & $\pi[\mathrm{Pa}]$ \\
\hline Fluid A & 3.0 & $7.499 \mathrm{e}+8$ \\
Fluid B & 1.4 & 0 \\
\hline
\end{tabular}

Table 6: Water-Air shock-interface interaction: Material parameters of the stiffened gas law for water and air. 


\begin{tabular}{|l|l|l|}
\hline & $W_{L}$ & $W_{R}$ \\
\hline$\rho\left[\mathrm{kg} / \mathrm{m}^{3}\right]$ & 0.9575 & 1000 \\
$u[\mathrm{~m} / \mathrm{s}]$ & 0 & 0 \\
$p[\mathrm{~Pa}]$ & $7.2568 \mathrm{e}+4$ & $1 \mathrm{e}+5$ \\
$\varphi$ & 1 & 0 \\
\hline
\end{tabular}

Table 7: Weakly compressed gas bubble: Initial left and right states. 


\begin{tabular}{|l|l|l|}
\hline & $\gamma[-]$ & $\pi[\mathrm{Pa}]$ \\
\hline Gas & 1.4 & 0 \\
Liquid & 3 & $7.333 \mathrm{e}+5$ \\
\hline
\end{tabular}

Table 8: Weakly compressed gas bubble: Material parameters. 


\begin{tabular}{|l|l|l|}
\hline & $W_{L}$ & $W_{R}$ \\
\hline$\rho\left[\mathrm{kg} / \mathrm{m}^{3}\right]$ & $9.575 \mathrm{e}-4$ & 1000 \\
$u[\mathrm{~m} / \mathrm{s}]$ & 0 & 0 \\
$p[\mathrm{~Pa}]$ & 4.58 & $1 \mathrm{e}+5$ \\
$\varphi$ & 1 & 0 \\
\hline
\end{tabular}

Table 9: Strongly compressed gas bubble: Initial left and right states. 


\begin{tabular}{|l|l|l|}
\hline & $\gamma[-]$ & $\pi[\mathrm{Pa}]$ \\
\hline Gas & 1.4 & 0 \\
Liquid & 3 & $7.499 \mathrm{e}+8$ \\
\hline
\end{tabular}

Table 10: Strongly compressed gas bubble: Material parameters. 